\title{
Parabolic conformally symplectic structures II: parabolic contactification
}

\author{
Andreas Čap ${ }^{1}$ (D) . Tomáš Salač ${ }^{2}$
}

Received: 31 January 2017 / Accepted: 22 November 2017 / Published online: 5 December 2017

(C) The Author(s) 2017. This article is an open access publication

\begin{abstract}
Parabolic almost conformally symplectic structures were introduced in the first part of this series of articles as a class of geometric structures which have an underlying almost conformally symplectic structure. If this underlying structure is conformally symplectic, then one obtains a PCS-structure. In the current article, we relate PCS-structures to parabolic contact structures. Starting from a parabolic contact structure with a transversal infinitesimal automorphism, we first construct a natural PCS-structure on any local leaf space of the corresponding foliation. Then we develop a parabolic version of contactification to show that any PCS-structure can be locally realized (uniquely up to isomorphism) in this way. In the second part of the paper, these results are extended to an analogous correspondence between contact projective structures and so-called conformally Fedosov structures. The developments in this article provide the technical background for a construction of sequences and complexes of differential operators which are naturally associated to PCS-structures by pushing down BGG sequences on parabolic contact structures. This is the topic of the third part of this series of articles.
\end{abstract}

Keywords Almost conformally symplectic structure - Special symplectic connection · Parabolic contact structure $\cdot$ Conformally Fedosov structure $\cdot$ Contactification

Mathematics Subject Classification Primary 53D05 $\cdot 53 \mathrm{D} 10 \cdot 53 \mathrm{~B} 15 \cdot 53 \mathrm{C} 10 \cdot 53 \mathrm{C} 15$; Secondary 53C29 $\cdot 53 \mathrm{C} 55$

Open access funding provided by Austrian Science Fund (FWF). Support by Projects P23244-N13 (both authors) and P27072-N25 (first author) of the Austrian Science fund (FWF) is gratefully acknowledged. We thank the referee for very helpful comments.

Andreas Čap

Andreas.Cap@univie.ac.at

Tomáš Salač

salac@karlin.mff.cuni.cz

1 Faculty of Mathematics, University of Vienna, Oskar-Morgenstern-Platz 1, 1090 Wien, Austria

2 Mathematical Institute, Charles University, Sokolovská 83, Praha, Czech Republic 


\section{Introduction}

This is the second part in a series of three articles devoted to the study of a class of geometric structures and of differential complexes naturally associated to these structures. The main motivation for this series are the examples of differential complexes on complex projective space, which were constructed and applied to questions of integral geometry in [9]. An attempt to put these complexes into the context of geometric structures was made in the first version of the preprint [8], which introduced the concept of a conformally Fedosov structure. The aim of providing a framework analogous to Bernstein-Gelfand-Gelfand resolutions (or BGG resolutions) associated to parabolic geometries was realized (for conformally Fedosov structures) in the second version of Eastwood and Slovák, [8], which has appeared recently.

Our article builds on [4], where we introduced a family of first-order structures, which all have an underlying almost conformally symplectic structure. There is one such structure for each contact grading of a simple Lie algebra, which is not of type $C_{n}$. These gradings are related to certain parabolic subalgebras and to parabolic contact structures as discussed below, which motivates the name parabolic almost conformally symplectic structures (or PACS-structures for short). The main result of Čap and Salač [4] is that each such structure determines a canonical connection on the tangent bundle, which is characterized by a normalization condition on its torsion. The torsion of this connection is a basic invariant of the structure, which naturally splits into two parts. One of these parts is the obstruction to the almost conformally symplectic structure being conformally symplectic, and requiring this part of the torsion to vanish, one arrives at the subclass of PCS-structures or parabolic conformally symplectic structures.

In this second part, we only consider PCS-structures and we relate them to another class of geometric structures, called parabolic contact structures. There is one such structure for each contact grading of a simple Lie algebra, and they all have an underlying contact structure. The most prominent example of a parabolic contact structure is provided by (partially integrable almost) CR-structures of hypersurface type. Via the relation to contact gradings, each type of PACS-structure determines a corresponding type of parabolic contact structure in one higher dimension.

Now for parabolic contact structures, there is the concept of transversal infinitesimal automorphisms, which in particular are transversal infinitesimal contactomorphisms for the underlying contact structure. Any transversal infinitesimal contactomorphism defines a foliation with one-dimensional leaves. In [6], we have shown that any local leaf space for such a foliation naturally inherits a conformally symplectic structure. Further we have shown that locally any conformally symplectic structure can be realized in this way and that these realizations are locally unique up to contactomorphism.

In the current article, we extend all these results to the setting of PCS-structures and parabolic contact structures, see Theorems 2, 4, and 5. Moreover, we discuss the relation between distinguished connections associated to parabolic contact structures and the canonical connections of PCS-structures. Together with Section 4.7 of [4], this allows us to complete the discussion of the relation of PCS-structures to special symplectic connections in the sense of Cahen and Schwachhöfer [3] and thus to exceptional holonomies, see Theorem 7.

The contact gradings of Lie algebras of type $C_{n}$ do not give rise to a PACS-structure. However, there is a type of parabolic contact structures associated to these gradings, the so-called contact projective structures. For the applications in [9] it was only necessary to construct the initial parts of certain differential complexes. However, the constructions can be extended, exhibiting that the results look similar to the BGG resolutions associated to 
locally flat contact projective structures. Also, the tractor bundle associated to a conformally Fedosov structure in [8] looks similar to the standard tractor bundle of a contact projective manifold in one higher dimension. So all that suggests that conformally Fedosov structures might be related to parabolic contact structures. In Sect. 3 of this article, we show that this is indeed the case and extend the above-mentioned results on structures on quotients and contactifications from Čap and Salač [6] also to this setting. This will allow us to include the $C_{n}$-types into a uniform treatment in the last part of this series.

There is also a class of special symplectic connections associated to Lie algebras of type $C_{n}$, the so-called symplectic connections of Ricci type, see [3] and [1]. We also get a characterization of these connections as conformally Fedosov structures with locally flat parabolic contactification, see Corollary 1 . Together with Theorem 7 , this recovers the fact that all special symplectic connections can be obtained from local quotients of the homogeneous models of parabolic contact structures, which is the crucial result of Cahen and Schwachhöfer [3].

A third important aim of this article is to give examples of global leaf spaces of compact contact manifolds which inherit PCS-structures, respectively, conformally Fedosov structures. From the point of view of differential complexes, such global quotients are interesting, since they lead to information on the cohomology of the resulting sequences, which are important for the applications in [9]. As one of these examples, we show that the Hopf fibration $S^{2 n+1} \rightarrow \mathbb{C} P^{n}$ can be considered as a global parabolic contactification in two ways. On the one hand, this concerns the CR-structure on $S^{2 n+1}$ coming from the embedding as a hypersurface in $\mathbb{C}^{n+1}$, which induces the Kähler structure on $\mathbb{C} P^{n}$ as the underlying PCSstructure. On the other hand, viewing $S^{2 n+1}$ as the space of real rays in $\mathbb{C}^{n+1}$ (viewed as a real symplectic vector space), it inherits a contact projective structure. This gives rise to the conformally Fedosov structure on $\mathbb{C} P^{n}$ studied in [8].

The second example of such a global space of leaves we discuss is a bit more exotic. In Theorem 3, we show that the Grassmannian of complex planes in $\mathbb{C}^{n+1}$ can be realized as a global space of leaves for a transversal infinitesimal automorphism of the space of those quaternionic lines in $\mathbb{H}^{n+1}$, which are isotropic for a quaternionic skew Hermitian form. This induces on the Grassmannian the PCS-structure of quaternionic type from Corollary 3.8 of [4], which is homogeneous under $S U(n+1)$ and can be viewed as a counterpart of the quaternion-Kähler metric on the Grassmannian of planes.

In the last part [5] of the series, we show how invariant differential operators for parabolic contact structures induce natural differential operators on PCS-quotients. There is a welldeveloped theory which in particular produces invariant differential complexes on locally flat parabolic contact structures and in certain curved situations. These can then be used to obtain invariant differential complexes associated to special symplectic connections as well as certain more general PCS-structures.

\section{Contactification of PCS-structures}

We start this section by briefly recalling the concept of PCS-structures as introduced in [4] and the class of parabolic contact structures. Then we describe the relation between the two kinds of structures, generalizing the analogous results from Čap and Salač [6], which relate conformally symplectic structures to contact structures. 


\subsection{Contact gradings and associated groups}

A contact grading on a simple Lie algebra $\mathfrak{g}$ over $\mathbb{K}=\mathbb{R}$ or $\mathbb{C}$ is a decomposition $\mathfrak{g}=$ $\mathfrak{g}_{-2} \oplus \mathfrak{g}_{-1} \oplus \mathfrak{g}_{0} \oplus \mathfrak{g}_{1} \oplus \mathfrak{g}_{2}$ which is compatible with the Lie bracket in the sense that $\left[\mathfrak{g}_{i}, \mathfrak{g}_{i}\right] \subset \mathfrak{g}_{i+j}$ (putting $\mathfrak{g}_{\ell}=\{0\}$ if $|\ell|>2$ ), such that $\operatorname{dim}\left(\mathfrak{g}_{-2}\right)=1$ and such that the bracket $\mathfrak{g}_{-1} \times \mathfrak{g}_{-1} \rightarrow \mathfrak{g}_{-2}$ is a non-degenerate bilinear form. It turns out that then the analogous statements hold for $\mathfrak{g}_{2}$ and the bracket $\mathfrak{g}_{1} \times \mathfrak{g}_{1} \rightarrow \mathfrak{g}_{2}$.

Such gradings are well known from the theory of quaternionic symmetric spaces as well as from the theory of parabolic contact structures. They exist on any complex simple Lie algebra of rank at least two and on most non-compact real simple Lie algebras (namely on those which contain a highest root vector). If they exist, they are always unique up to isomorphism. The complete list of contact gradings can be found in Example 3.2.10 of [7].

Via $\mathfrak{g}_{0} \subset \mathfrak{g}$, which is a Lie subalgebra by the grading property, contact gradings give rise to geometric structures in two ways. On the one hand, consider the graded Lie algebra $\mathfrak{g}_{-}:=\mathfrak{g}_{-2} \oplus \mathfrak{g}_{-1}$ which, by definition of a contact grading, is a Heisenberg algebra. By the grading property and the Jacobi-identity, the adjoint action of any element $A \in \mathfrak{g}_{0}$ can be restricted to $\mathfrak{g}_{-}$and defines a derivation on this Lie algebra which preserves the grading. It is easy to see that the resulting Lie algebra homomorphism $\mathfrak{g}_{0} \rightarrow \mathfrak{d} \mathfrak{e} \mathfrak{r}_{\mathrm{gr}}\left(\mathfrak{g}_{-}\right)$is always injective.

It is easy to describe $\mathfrak{d e r} \mathfrak{r}_{\text {gr }}\left(\mathfrak{g}_{-}\right)$explicitly. Viewing the bracket as a linear map $\Lambda^{2} \mathfrak{g}_{-1} \rightarrow$ $\mathfrak{g}_{-2}$, its kernel defines a codimension-one subspace $\Lambda_{0}^{2} \mathfrak{g}_{-1} \subset \Lambda^{2} \mathfrak{g}_{-1}$. Now one easily shows that a linear map $\varphi: \mathfrak{g}_{-1} \rightarrow \mathfrak{g}_{-1}$ can be extended to a derivation of $\mathfrak{g}_{-}$if and only if the induced endomorphism of $\Lambda^{2} \mathfrak{g}_{-1}$ preserves the hyperplane $\Lambda_{0}^{2} \mathfrak{g}_{-1}$ and thus factors to an endomorphism of the quotient which is isomorphic to $\mathfrak{g}_{-2}$. The bracket defines a nondegenerate bilinear form on $\mathfrak{g}_{-1}$ up to scale and thus a line in $\Lambda^{2}\left(\mathfrak{g}_{-1}\right)^{*}$, which is exactly the annihilator of $\Lambda_{0}^{2} \mathfrak{g}_{-1}$. The stabilizer of this line in $L\left(\mathfrak{g}_{-1}, \mathfrak{g}_{-1}\right)$ will be denoted by $\mathfrak{c} \mathfrak{s}\left(\mathfrak{g}_{-1}\right)$ and called the conformally symplectic Lie algebra of $\mathfrak{g}_{-1}$. The above discussion implies that the adjoint action of $\mathfrak{g}_{0}$ on $\mathfrak{g}_{-1}$ defines an injective homomorphism $\mathfrak{g}_{0} \hookrightarrow \mathfrak{c s p}\left(\mathfrak{g}_{-1}\right)$.

The case of algebras of type $C_{n}$ is special with respect to contact gradings. In this case $\mathfrak{g}$ itself is a symplectic Lie algebra and it turns out that the adjoint action actually gives rise to an isomorphism $\mathfrak{g}_{0} \cong \mathfrak{c s p}\left(\mathfrak{g}_{-1}\right) \cong \mathfrak{d e r}_{g r}\left(\mathfrak{g}_{-}\right)$, so one just obtains the full conformally symplectic algebra in this case. Therefore, we will have to exclude the algebras of type $C_{n}$ from the considerations in the rest of Sect. 2. Conformally Fedosov structures and their contactifications, which will be discussed in Sect. 3, can be thought of as a $C_{n}$-analog of the situation discussed here.

To proceed toward geometric structures determined by a contact grading, we also need a choice of group associated to the Lie algebra $\mathfrak{g}_{0}$. While the theory of PACS-structures can be developed for more general Lie groups with Lie algebra $\mathfrak{g}_{0}$, we will restrict the choice of group here to get a complete correspondence to parabolic contact structures. Namely, let us start with a Lie group $G$ with Lie algebra $\mathfrak{g}$. Then it is well known that the Lie subalgebra $\mathfrak{p}:=\mathfrak{g}_{0} \oplus \mathfrak{g}_{1} \oplus \mathfrak{g}_{2} \subset \mathfrak{g}$ is a parabolic subalgebra, so in particular, the normalizer of $\mathfrak{p}$ in $G$ is a Lie subgroup with Lie algebra $\mathfrak{p}$. Let $P \subset G$ be a parabolic subgroup of $G$ corresponding to $\mathfrak{p}$, i.e., a subgroup lying between this normalizer and its connected component of the identity. Then $P$ has Lie algebra $\mathfrak{p}$ and it is well known that the adjoint action of any element of $P$ maps each $\mathfrak{g}_{i}$ to $\mathfrak{g}_{i} \oplus \cdots \oplus \mathfrak{g}_{2}$, so it preserves the filtration of $\mathfrak{g}$ induced by the grading.

Finally, one defines a Lie subgroup $G_{0} \subset P$ as consisting of those elements whose adjoint action preserves the grading on $\mathfrak{g}$. Let us denote by $C S p\left(\mathfrak{g}_{-1}\right) \subset G L\left(\mathfrak{g}_{-1}\right)$ the subgroup of those linear isomorphisms for which the induced automorphism of $\Lambda^{2}\left(\mathfrak{g}_{-1}\right)^{*}$ preserves the line determined by the bracket. Then this can be identified with the group $\operatorname{Aut}_{g r}\left(\mathfrak{g}_{-}\right)$of 
automorphisms of the graded Lie algebra $\mathfrak{g}_{-}$and the adjoint action defines an infinitesimally injective homomorphism $G_{0} \rightarrow C S p\left(\mathfrak{g}_{-1}\right)$.

\subsection{PCS-structures}

Having constructed the infinitesimally injective homomorphism $G_{0} \rightarrow C S p\left(\mathfrak{g}_{-1}\right) \subset$ $G L\left(\mathfrak{g}_{-1}\right)$, there is the natural notion of a first-order structure with structure group $G_{0}$ on smooth manifolds of dimension $\operatorname{dim}\left(\mathfrak{g}_{-1}\right)$. These are the PACS-structures as introduced in [4]. The simplest way to describe such a structure is as a principal bundle $p: \mathcal{G}_{0} \rightarrow M$ with structure group $G_{0}$, which is endowed with a strictly horizontal, $G_{0}$-equivariant one-form $\theta \in \Omega^{1}\left(\mathcal{G}_{0}, \mathfrak{g}_{-1}\right)$. Here equivariancy means that for the principal right action $r^{g}$ of $g \in G_{0}$, one has $\left(r^{g}\right)^{*} \theta=\operatorname{Ad}\left(g^{-1}\right) \circ \theta$ while strict horizontality means that in each point $u \in \mathcal{G}_{0}$, the kernel of $\theta(u): T_{u} \mathcal{G}_{0} \rightarrow \mathfrak{g}_{-1}$ coincides with the vertical subbundle of $\mathcal{G}_{0} \rightarrow M$. In particular, $\theta(u)$ descends to a linear isomorphism $T_{p(u)} M \rightarrow \mathfrak{g}_{-1}$ which identifies $\mathcal{G}_{0}$ as a reduction of the linear frame bundle of $T M$.

Given $\left(\mathcal{G}_{0} \rightarrow M, \theta\right)$ as above, a representation of $G_{0}$ on a vector space $V$ gives rise to the natural vector bundle $\mathcal{G}_{0} \times{ }_{G_{0}} V \rightarrow M$. Via $\theta$, the bundle $\mathcal{G}_{0} \times{ }_{G_{0}} \mathfrak{g}_{-1}$ is identified with $T M$. Further, the $G_{0}$-invariant line in $\Lambda^{2}\left(\mathfrak{g}_{-1}\right)^{*}$ gives rise to a line subbundle $\ell \subset \Lambda^{2} T^{*} M$ such that each nonzero element of $\ell$ is non-degenerate as a bilinear form on the corresponding tangent space. This is the almost conformally symplectic structure underlying a PACS-structure. While the theory of PACS-structures is developed in this general setting in [4], we will impose a restriction on such structures throughout this article. Namely, we will only deal with PCS-structures, i.e., assume that the underlying structure is conformally symplectic. This means that the line subbundle $\ell$ admits local sections which are closed as two-forms on $M$.

A central result of Čap and Salač [4] is that any PACS-structure on $M$ determines a canonical linear connection on $T M$, whose torsion satisfies a certain normalization condition. We formulate this result only for PCS-structures, where the normalization condition on the torsion is simpler. To formulate this condition, we need the fact that there is a natural $G_{0}$-invariant subspace $\operatorname{ker}(\square) \subset \Lambda^{2}\left(\mathfrak{g}_{-1}\right)^{*} \otimes \mathfrak{g}_{-1}$, called the harmonic subspace. Here $\square: \Lambda^{2}\left(\mathfrak{g}_{-}\right)^{*} \otimes \mathfrak{g}_{-} \rightarrow \Lambda^{2}\left(\mathfrak{g}_{-}\right)^{*} \otimes \mathfrak{g}_{-}$is the Kostant-Laplacian, see Section 4.3 of [4] for the definition and Sections 4.5 and 4.6 of this reference as well as Section 4.2 of [7] for an explicit description of this subspace in several cases. Via associated bundles, this gives rise to a subbundle $\operatorname{ker}(\square) \subset \Lambda^{2} T^{*} M \otimes T M$, whose elements are called algebraically harmonic. Specialized to the PCS-case, Corollary 4.3 of [4] shows

Theorem 1 Let $\left(\mathcal{G}_{0} \rightarrow M, \theta\right)$ be a PCS-structure (associated to a contact grading on a simple Lie algebra which is not of type $C_{n}$ ) on a smooth manifold $M$. Then there is a unique connection compatible with this structure, such that the induced linear connection on TM has algebraically harmonic torsion.

\subsection{Parabolic contact structures}

There is a second way to obtain a geometric structure from a contact grading of a real simple Lie algebra $\mathfrak{g}$. Consider a smooth manifold $M^{\#}$ of $\operatorname{dimension} \operatorname{dim}\left(\mathfrak{g}_{-}\right)=\operatorname{dim}\left(\mathfrak{g}_{-1}\right)+1$ and suppose that $H \subset T M^{\#}$ is a smooth distribution of corank one. Denoting by $Q$ the quotient bundle $T M^{\#} / H$ (which is a real line bundle), the Lie bracket of vector fields induces a skew symmetric bilinear bundle map $\mathcal{L}: H \times H \rightarrow Q$ called the Levi bracket. Now $H$ is a contact structure if and only if this Levi bracket is non-degenerate in each point $x \in M^{\#}$. Equivalently, for each point $x$, the associated graded $H_{x} \oplus Q_{x}$ of the tangent space $T_{x} M^{\#}$ endowed with 
$\mathcal{L}_{x}$ as a Lie bracket is isomorphic to $\mathfrak{g}_{-}$. Supposing that $H \subset T M^{\#}$ is a contact structure, it is then easy to obtain an adapted frame bundle for the associated graded vector bundle $\operatorname{gr}\left(T M^{\#}\right)$ to the filtered vector bundle $H \subset T M^{\#}$ with structure group $\operatorname{Aut}_{g r}\left(\mathfrak{g}_{-}\right) \cong C \operatorname{Sp}\left(\mathfrak{g}_{-1}\right)$.

If $\mathfrak{g}$ is not of type $C_{n}$, then a parabolic contact structure of the corresponding type can be simply described as a reduction of structure group of this adapted frame bundle corresponding to the infinitesimally injective homomorphism $G_{0} \rightarrow \operatorname{Aut}_{g r}\left(\mathfrak{g}_{-}\right)$defined by the adjoint action. Similarly to the case of standard first-order structures, such a reduction can be described as an abstract principal $G_{0}$-bundle $p_{0}^{\#}: \mathcal{G}_{0}^{\#} \rightarrow M^{\#}$ endowed with an analog of a soldering form as follows.

Define $T^{-1} \mathcal{G}_{0}^{\#} \subset T \mathcal{G}_{0}^{\#}$ as the preimage of $H \subset T M^{\#}$ under the natural projection. Of course, this contains the vertical subbundle $V \mathcal{G}_{0}^{\#}=\operatorname{ker}\left(T p_{0}^{\#}\right)$. Then the soldering form consists of two components, namely $\theta_{-2}^{\#} \in \Omega^{1}\left(\mathcal{G}_{0}^{\#}, \mathfrak{g}_{-2}\right)$ and $\theta_{-1}^{\#} \in \Gamma\left(L\left(T^{-1} \mathcal{G}_{0}^{\#}, \mathfrak{g}_{-1}\right)\right)$. So while $\theta_{-2}^{\#}$ is an ordinary differential form, $\theta_{-1}^{\#}$ is only defined on the subbundle $T^{-1} \mathcal{G}_{0}^{\#}$. Both components are required to be $G_{0}$-equivariant (with respect to the adjoint action), and they should be strictly horizontal in the sense that for each $u \in \mathcal{G}_{0}^{\#}$ one has $\operatorname{ker}\left(\theta_{-2}^{\#}(u)\right)=$ $T_{u}^{-1} \mathcal{G}_{0}^{\#}$ and $\operatorname{ker}\left(\theta_{-1}^{\#}(u)\right)=V_{u} \mathcal{G}_{0}^{\#}$. This implies that $\theta_{-2}^{\#}(u)$ descends to a linear isomorphism $Q_{p_{0}^{\#}(u)} \rightarrow \mathfrak{g}_{-2}$ while $\theta_{-1}^{\#}(u)$ descends to a linear isomorphism $H_{p_{0}^{\#}(u)} \rightarrow \mathfrak{g}_{-1}$. These isomorphisms together define an isomorphism of Lie algebras, i.e., they intertwine between the Levi bracket and the Lie bracket on $\mathfrak{g}_{-}$.

Parabolic contact structures admit an equivalent uniform description, which also works in the $C_{n}$-case. Namely, given a smooth manifold $M^{\#}$ as above, one considers a principal bundle $p^{\#}: \mathcal{G}^{\#} \rightarrow M^{\#}$ with structure group $P$, which is endowed with a Cartan connection $\omega \in$ $\Omega^{1}\left(\mathcal{G}^{\#}, \mathfrak{g}\right)$. This by definition means that $\omega$ is $P$-equivariant and reproduces the generators of fundamental vector fields, and that for each $u \in \mathcal{G}^{\#}$ the map $\omega(u): T_{u} \mathcal{G}^{\#} \rightarrow \mathfrak{g}$ is a linear isomorphism. Moreover, one has to assume the conditions of regularity and normality on the curvature of the Cartan connection $\omega$, see Section 3.1 of [7] for the precise definitions.

The two descriptions are related as follows. Given the Cartan geometry $\left(\mathcal{G}^{\#}, \omega\right)$ and a point $u \in \mathcal{G}^{\#}$ with $x=p^{\#}(u) \in M^{\#}, \omega(u)$ descends to a linear isomorphism $T_{x} M^{\#} \rightarrow \mathfrak{g} / \mathfrak{p}$. The subset $\mathfrak{g}_{-1} \oplus \mathfrak{p} \subset \mathfrak{g}$ is $P$-invariant so its preimage gives rise to a well-defined subspace $H_{x} \subset T_{x} M^{\#}$. These subspaces fit together to a corank one subbundle $H \subset T M^{\#}$ which defines a contact structure by regularity of $\omega$. Moreover, the exponential mapping restricts to a diffeomorphism from $\mathfrak{p}_{+}:=\mathfrak{g}_{1} \oplus \mathfrak{g}_{2}$ onto a closed normal subgroup $P_{+} \subset P$, such that $G_{0} \cong P / P_{+}$via the restriction of the canonical projection. The group $P_{+}$acts freely on $\mathcal{G}^{\#}$ via the principal right action, and the quotient $\mathcal{G}_{0}^{\#}:=\mathcal{G}^{\#} / P_{+}$naturally is a principal bundle over $M^{\#}$ with structure group $P / P_{+}=G_{0}$. Finally, one shows that the component of $\omega$ in $\mathfrak{g}_{-2}$ descends to $\theta_{-2}^{\#} \in \Omega^{1}\left(\mathcal{G}_{0}^{\#}, \mathfrak{g}_{-2}\right)$ while an appropriate restriction of the $\mathfrak{g}_{-1}$-component of $\omega$ descends to $\theta_{-1}^{\#} \in \Gamma\left(L\left(T^{-1} \mathcal{G}_{0}^{\#}, \mathfrak{g}_{-1}\right)\right)$.

In the $C_{n}$-case, the underlying structure constructed in this way is just the adapted frame bundle of a contact structure on $M^{\#}$ and does not contain any additional information. In all other cases, however, the Cartan geometry $\left(\mathcal{G}^{\#}, \omega\right)$ can be reconstructed from the underlying structure by a prolongation procedure as described in Section 3.1 of [7]. One forms the obvious extension $\mathcal{G}_{0}^{\#} \times{ }_{G_{0}} P$ of structure group and then shows that there is a normal Cartan connection on this principal bundle inducing the given soldering form. Further, one proves that any isomorphism of underlying structures lifts to an isomorphism of normal Cartan geometries, which establishes an equivalence (in the categorical sense) between the two pictures. 


\subsection{PCS-quotients and parabolic contactifications}

Recall that for a contact manifold $\left(M^{\#}, H\right)$ an infinitesimal contactomorphism is a vector field $\xi \in \mathfrak{X}\left(M^{\#}\right)$ such that for all $\eta \in \Gamma(H)$ we get $[\xi, \eta] \in \Gamma(H)$. An infinitesimal contactomorphism is called transversal if $\xi(x) \notin H_{x}$ for all $x \in M^{\#}$. This in particular implies that $\xi$ is nowhere vanishing and thus defines a foliation of $M^{\#}$ with one-dimensional leaves. By a quotient of $M^{\#}$ by $\xi$ one then means a global space of leaves, i.e., a surjective submersion $q: M^{\#} \rightarrow M$ with connected fibers such that for each $x \in M^{\#}$ the vertical subspace $\operatorname{ker}\left(T_{x} q\right) \subset T_{x} M^{\#}$ coincides with the line spanned by $\xi(x)$.

In this situation, Proposition 2.2 of [6] shows that the contact structure on $M^{\#}$ induces a conformally symplectic structure $\ell \subset \Lambda^{2} T^{*} M$. Denoting by $\alpha$ the unique contact form on $M^{\#}$ such that $\alpha(\xi)=1$, there even is a global symplectic form $\omega$ on $M$, which is a section of $\ell$ and such that $q^{*} \omega=d \alpha$. Conversely, given a manifold $M$ endowed with a conformally symplectic structure $\ell$, one can try to (locally) realize it as a quotient of a contact manifold $\left(M^{\#}, H\right)$, which then is called a (local) contactification of $(M, \ell)$. Existence and uniqueness of such contactifications is established in [6].

We want to develop refinements of these concepts which relate parabolic contact structures to PCS-structures. Let us start with the regular normal parabolic geometry $\left(p: \mathcal{G}^{\#} \rightarrow M^{\#}, \omega\right)$ of type $(G, P)$ corresponding to a parabolic contact structure with contact distribution $H \subset$ $T M^{\#}$. An infinitesimal symmetry of the geometry then is a vector field $\tilde{\xi} \in \mathfrak{X}\left(\mathcal{G}^{\#}\right)$ which is $P$-invariant and satisfies $L_{\tilde{\xi}} \omega=0$, where $L$ denotes the Lie derivative. A $P$-invariant vector field is automatically projectable, so there is a corresponding vector field $\xi \in \mathfrak{X}\left(M^{\#}\right)$ as well as an intermediate $G_{0}$-invariant vector field $\xi_{0} \in \mathfrak{X}\left(\mathcal{G}_{0}^{\#}\right)$. It is easy to verify directly that $\xi$ is an infinitesimal contactomorphism. As before, an infinitesimal symmetry is called transverse if $\xi(x) \notin H_{x}$ for all $x \in M^{\#}$.

In the case that $\mathfrak{g}$ is not of type $C_{n}$, infinitesimal symmetries can be equivalently described in terms of the underlying structure $\left(p_{0}^{\#}: \mathcal{G}_{0}^{\#} \rightarrow M^{\#}, \theta^{\#}\right)$. For a $G_{0}$-invariant vector field $\xi_{0} \in \mathfrak{X}\left(\mathcal{G}_{0}^{\#}\right)$ to be an infinitesimal symmetry, one first has to require that $\left[\xi_{0}, \eta\right] \in \Gamma\left(T^{-1} \mathcal{G}_{0}^{\#}\right)$ for all $\eta \in \Gamma\left(T^{-1} \mathcal{G}_{0}^{\#}\right)$ or equivalently that the projection $\xi \in \mathfrak{X}\left(M^{\#}\right)$ of $\xi_{0}$ is an infinitesimal contactomorphism. If this is the case, there is a well-defined Lie derivative $L_{\xi_{0}} \theta_{-1}^{\#}$ of the partially defined differential form $\theta_{-1}^{\#}$, and $\xi_{0}$ is an infinitesimal symmetry if $L_{\xi_{0}} \theta_{i}^{\#}=0$ for $i=-1,-2$.

Definition 1 Consider a parabolic contact structure of type $(G, P)$ with underlying structure $\left(p_{0}^{\#}: \mathcal{G}_{0}^{\#} \rightarrow M^{\#}, \theta^{\#}\right)$ and a transversal infinitesimal symmetry $\xi_{0} \in \mathfrak{X}\left(\mathcal{G}_{0}^{\#}\right)$ of this structure. Then a PCS-quotient of the parabolic contact structure is a PCS-structure $\left(p_{0}: \mathcal{G}_{0} \rightarrow M, \theta\right)$ with structure group $G_{0}$ together with a morphism $q_{0}: \mathcal{G}_{0}^{\#} \rightarrow \mathcal{G}_{0}$ of principal bundles such that

- $q_{0}$ is a surjective submersion with connected fibers.

- For each $u^{\#} \in \mathcal{G}_{0}^{\#}$ the kernel of $T_{u^{\#}} q_{0}$ is spanned by $\xi_{0}\left(u^{\#}\right)$.

- The restriction of $q_{0}^{*} \theta \in \Omega^{1}\left(\mathcal{G}_{0}^{\#}, \mathfrak{g}_{-1}\right)$ to elements of $T^{-1} \mathcal{G}_{0}^{\#}$ coincides with $\theta_{-1}^{\#}$.

In this situation, ( $\left.p_{0}^{\#}: \mathcal{G}_{0}^{\#} \rightarrow M^{\#}, \theta^{\#}, \xi_{0}\right)$ is also referred to as a parabolic contactification of the PCS-structure $\left(p_{0}: \mathcal{G}_{0} \rightarrow M, \theta\right)$.

To study these concepts, we need some preliminary observations. Consider the underlying structure $\left(p_{0}^{\#}: \mathcal{G}_{0}^{\#} \rightarrow M^{\#}, \theta^{\#}\right)$ of a parabolic contact structure of type $(G, P)$, a transverse symmetry $\xi_{0} \in \mathfrak{X}\left(\mathcal{G}_{0}^{\#}\right)$ of this structure and the projected vector field $\xi \in \mathfrak{X}\left(M^{\#}\right)$. Then both $\xi_{0}$ and $\xi$ are nowhere vanishing and hence define foliations with one-dimensional leaves. 
Lemma 1 In this situation, we have:

1. If $N \subset \mathcal{G}_{0}^{\#}$ is a leaf of the foliation defined by $\xi_{0}$, then $p_{0}^{\#}(N) \subset M^{\#}$ is a leaf of the foliation defined by $\xi$ and the restriction of $p_{0}^{\#}$ to $N$ is a covering map $N \rightarrow p_{0}^{\#}(N)$.

2. Suppose that there is a PCS-structure $\left(p_{0}: \mathcal{G}_{0} \rightarrow M, \theta\right)$ and a PCS-quotient $q_{0}$ : $\mathcal{G}_{0}^{\#} \rightarrow \mathcal{G}_{0}$ by $\xi_{0}$. Then the base map $q: M^{\#} \rightarrow M$ of $q_{0}$ is a quotient by the transverse infinitesimal contactomorphism $\xi$. Moreover, in this case the covering from (1) actually have to be diffeomorphisms.

Proof 1. Since $\xi_{0}$ projects to $\xi$ and is transversal to the vertical subbundle of $p_{0}^{\#}$, it is clear that $p_{0}^{\#}$ maps small open subsets of $N$ to integral submanifolds of the distribution spanned by $\xi$. Hence $p_{0}^{\#}(N)$ is a connected immersed integral manifold for the foliation defined by $\xi$ and thus contained in some leaf $\underline{N}$ of this foliation. Again by construction, $\left.p_{0}^{\#}\right|_{N}: N \rightarrow \underline{N}$ has bijective tangent maps in all points, so it is a local diffeomorphism and hence $p_{0}^{\#}(N) \subset \underline{N}$ is open.

Next, we observe that $\xi_{0}$ is $G_{0}$-equivariant. Hence if the flow of $\xi_{0}$ through a point $u^{\#}$ is defined on some time interval around zero, the same is true for $u^{\#} \cdot g$ for each $g \in G_{0}$. Now each such local flow line is either contained in $N$ or disjoint from it. In particular, for $u^{\#} \in N$, such a local flow line projects to an open neighborhood of $p_{0}^{\#}\left(u^{\#}\right)$ in $\underline{N}$, whose preimage in $N$ is the disjoint union of those flow lines, which are contained in $N$. By definition, these are open in the manifold topology of $N$.

On the other hand, if $x^{\#} \in \underline{N}$ lies in the closure of $p_{0}^{\#}(N)$, then choose $u^{\#} \in \mathcal{G}_{0}^{\#}$ lying over $x^{\#}$. For a local integral curve $c$ for $\xi_{0}$ through $u^{\#}, p_{0}^{\#} \circ c$ fills an open subset of $\underline{N}$ and hence intersects $p_{0}^{\#}(N)$. Shifting $c$ by the principal right action of an appropriate element of $G_{0}$, we may assume that $c$ intersects $N$, and hence is contained in $N$. Thus $x^{\#} \in p_{0}^{\#}(N)$, so $p_{0}^{\#}(N) \subset N$ is closed and hence $p_{0}^{\#}(N)=\underline{N}$. Since we have constructed trivializing neighborhoods for $p_{0}^{\#}: N \rightarrow \underline{N}$ already, this completes the proof of 1 .

2. By assumption, we have $p_{0} q_{0}=q \circ p_{0}^{\#}$. Since both $p_{0}$ and $q_{0}$ are surjective submersions, we see that $q$ is a surjective submersion. Since $\xi_{0}$ projects to $\xi$, it is also clear that the values of $\xi$ lie in $\operatorname{ker}(T q)$ and since each of these kernels has to be one-dimensional, it is spanned by the value of $\xi$. Now consider a fiber $N$ of $q_{0}$. Then $p_{0}^{\#}(N)$ lies in one fiber of $q$ and using $G_{0}$-equivariancy of $q_{0}$, one easily verifies that $p_{0}^{\#}(N)$ is all of this fiber. Hence the fibers of $q$ are connected, so the first statement is proved. But now the fibers of $q_{0}$ and $q$ are closed connected integral submanifolds for the distributions defined by $\xi_{0}$ and $\xi$, and hence have to coincide with the leaves. But since $q_{0}$ is a morphism of principal bundles, its restriction to each fiber of $p_{0}^{\#}$ is injective, so $p_{0}^{\#}$ is injective on fibers of $q_{0}$, and the last claim follows.

\subsection{Existence of PCS-quotients}

Part 2 of Lemma 1 tells us how to naturally phrase the question of existence of PCS-quotients. We assume that we start with a parabolic contact structure on $M^{\#}$ and the underlying vector field $\xi \in \mathfrak{X}\left(M^{\#}\right)$ of an infinitesimal symmetry of this structure. Then the natural question to ask is when a quotient $q: M^{\#} \rightarrow M$ of $M^{\#}$ by $\xi$ can be made into a PCS-quotient. We can now show that the necessary condition from part 2 of Lemma 1 is also sufficient.

Theorem 2 Consider a parabolic contact structure of type $(G, P)$ with $G$ not of type $C_{n}$ on a smooth manifold $M^{\#}$ with underlying $G_{0}$-bundle $p_{0}^{\#}: \mathcal{G}_{0}^{\#} \rightarrow M^{\#}$. Let $\xi_{0} \in \mathfrak{X}\left(\mathcal{G}_{0}^{\#}\right)$ be a transverse infinitesimal symmetry of this structure and let $\xi \in \mathfrak{X}\left(M^{\#}\right)$ be the underlying 
infinitesimal contactomorphism. Suppose that for each leaf $N \subset \mathcal{G}_{0}^{\#}$ of the foliation defined by $\xi_{0}$, the restriction $\left.p_{0}^{\#}\right|_{N}: N \rightarrow p_{0}^{\#}(N)$ is a diffeomorphism.

Then for any quotient $q: M^{\#} \rightarrow M$ by $\xi$, there is a canonical PCS-structure $\mathcal{G}_{0} \rightarrow M$ on $M$ such that $q$ becomes a PCS-quotient.

Proof Let $\sim$ be the equivalence relation on $\mathcal{G}_{0}^{\#}$ defined by the foliation induced by $\xi_{0}$, i.e., two points are equivalent if they lie in the same leaf. Define $\mathcal{G}_{0}:=\mathcal{G}_{0}^{\#} / \sim$, the set of equivalence classes, and let $q_{0}: \mathcal{G}_{0}^{\#} \rightarrow \mathcal{G}_{0}$ be the canonical map. From the proof of Lemma 1, we know that $p_{0}^{\#}$ maps leaves to leaves, and the leaves in $M^{\#}$ are the fibers of $q$, so there is a set map $p_{0}: \mathcal{G}_{0} \rightarrow M$ such that $p_{0} \circ q_{0}=q \circ p_{0}^{\#}$.

We claim that $p_{0}: \mathcal{G}_{0} \rightarrow M$ is a $G_{0}$-principal bundle and $q_{0}$ is a morphism of principal bundles. To prove this, observe first that $q \circ p_{0}^{\#}$ is a surjective submersion. Hence for each $x \in M$, there is a neighborhood $U$ of $x$ in $M$ and a smooth map $\tau: U \rightarrow \mathcal{G}_{0}^{\#}$ such that $q \circ p_{0}^{\#} \circ \tau=\mathrm{id}_{U}$. Using this, we define a map $\psi: U \times G_{0} \rightarrow \mathcal{G}_{0}$ by $\psi(y, g):=q_{0}(\tau(y) \cdot g)$. This evidently satisfies $p_{0} \circ \psi=\operatorname{pr}_{1}$, so it has values in $p_{0}^{-1}(U)$. If $\psi(y, g)=\psi(\tilde{y}, \tilde{g})$, then applying $p_{0}$ we get $y=\tilde{y}$. Moreover, $\tau(y) \cdot g$ and $\tau(y) \cdot \tilde{g}$ lie in the same leaf in $\mathcal{G}_{0}^{\#}$. Since we have assumed that $p_{0}^{\#}$ restricts to an injection on each leaf, we conclude that $\tau(y) \cdot g=\tau(y) \cdot \tilde{g}$ and thus $g=\tilde{g}$. On the other hand, for $y \in U$, a point $z$ in $p_{0}^{-1}(U)$ corresponds to a leaf $N \subset \mathcal{G}_{0}^{\#}$ such that $p_{0}^{\#}(N)=q^{-1}(\{y\})$. Hence there is a point $z^{\#} \in N$ such that $p_{0}^{\#}\left(z^{\#}\right)=p_{0}^{\#}(\tau(y)) \in q^{-1}(\{y\})$. But this implies $z^{\#}=\tau(y) \cdot g$ for some $g \in G_{0}$ and hence $z=\psi(y, g)$, so $\psi: U \times G_{0} \rightarrow p_{0}^{-1}(U)$ is bijective.

Now suppose that for some open subset $U \subset M$ we have two sections $\tau$ and $\hat{\tau}$ as above, and let us consider the "chart change" $\varphi: U \times G_{0} \rightarrow G_{0}$, i.e., the map characterized by $q_{0}(\tau(x) \cdot g)=q_{0}(\hat{\tau}(x) \cdot \varphi(x, g))$ (which evidently exists). It suffices to do this locally around a fixed point $x$. By construction $p_{0}^{\#}(\tau(x))$ and $p_{0}^{\#}(\hat{\tau}(x))$ both lie in the leaf $q^{-1}(x)$, so there is a time $t_{0} \in \mathbb{R}$ such that $p_{0}^{\#}(\tau(x))=\mathrm{Fl}_{t_{0}}^{\xi}\left(p_{0}^{\#}(\hat{\tau}(x))\right)$. It is easy to see that the flow $\mathrm{Fl}_{t_{0}}^{\xi_{0}}$ is defined in the point $\hat{\tau}(x)$. Thus, we may shrink $U$ in such a way that for some $\epsilon>0$, $\mathrm{Fl}_{t_{0}+t}^{\xi_{0}}(\hat{\tau}(y))$ is defined for all $y \in U$ if $|t|<\epsilon$, and moreover $(y, t) \mapsto p_{0}^{\#}\left(\mathrm{Fl}_{t_{0}+t}^{\xi_{0}}(\hat{\tau}(y))\right)$ is a diffeomorphism from $U \times(-\epsilon, \epsilon)$ onto an open neighborhood $U^{\#}$ of $p_{0}^{\#}(\tau(x))$ in $M^{\#}$. Then there is an open neighborhood $V$ of $x$ in $M$ such that $p_{0}^{\#}(\tau(V)) \subset U^{\#}$, and we obtain a smooth function $\beta: V \rightarrow(-\epsilon, \epsilon)$ such that $p_{0}^{\#}(\tau(y))=p_{0}^{\#}\left(\mathrm{Fl}_{t_{0}+\beta(y)}^{\xi_{0}} \hat{\tau}(y)\right)$.

Now observe that $\tilde{\tau}(y):=\mathrm{Fl}_{t_{0}+\beta(y)}^{\xi_{0}}(\hat{\tau}(y))$ is a smooth section of $q \circ p_{0}^{\#}$ on $V$ which produces the same map $\psi$ as $\hat{\tau}$. But now $p_{0}^{\#} \circ \tilde{\tau}=p_{0}^{\#} \circ \tau$, so there is a smooth map $\gamma: V \rightarrow G_{0}$ such that $\tilde{\tau}(y)=\tau(y) \cdot \gamma(y)$, and hence $q_{0}(\tilde{\tau}(y) \cdot g)=q_{0}(\tau(y) \cdot(\gamma(y) g))$. This shows that we can endow $\mathcal{G}_{0}$ with a topology by requiring that the maps $\psi$ are homeomorphisms, and then there inverses define principal bundle charts, so the claim is proved.

By construction, the quotient $q: M^{\#} \rightarrow M$ has the property that for each $x \in M^{\#}$, the tangent map $T_{x} q$ restricts to a linear isomorphism $H_{x} \rightarrow T_{q(x)} M$. Hence for each $u \in \mathcal{G}_{0}^{*}$ the tangent map $T_{u} q_{0}$ restricts to a linear isomorphism $T_{u}^{-1} \mathcal{G}_{0}^{\#} \rightarrow T_{q_{0}(u)} \mathcal{G}_{0}$, which in addition respects the vertical subbundles. Composing $\theta_{-1}^{\#}(u)$ with the inverse of this isomorphism, we obtain a surjective linear map $T_{q_{0}(u)} \mathcal{G}_{0} \rightarrow \mathfrak{g}_{-1}$ whose kernel is the vertical subspace $V_{q_{0}(u)} \mathcal{G}_{0}$. Since $\theta_{-1}^{\#}$ is preserved by the flow of $\xi_{0}$, it follows that locally around $u$ points in the same fiber lead to the same map on $T_{q_{0}(u)} \mathcal{G}_{0}$. Since the fibers of $q_{0}$ are connected, we obtain a well-defined map $\theta\left(q_{0}(u)\right): T_{q_{0}(u)} \mathcal{G}_{0} \rightarrow \mathfrak{g}_{-1}$ with kernel the vertical subspace.

Now $\operatorname{ker}\left(T q_{0}\right) \subset T \mathcal{G}_{0}^{\#}$ is a smooth subbundle which is complementary to $T^{-1} \mathcal{G}_{0}^{\#}$, so we can use this to define a projection $\Pi: T \mathcal{G}_{0}^{\#} \rightarrow T^{-1} \mathcal{G}_{0}^{\#}$. Given $\eta \in \mathfrak{X}\left(\mathcal{G}_{0}\right)$, we can use a local smooth section $\sigma$ of $q_{0}$ to write $\theta(\eta)$ as $\theta_{-1}^{\#}(\Pi(T \sigma \circ \eta))$, which shows that $\theta$ is smooth. 
Finally $\theta$ is strictly horizontal by construction and $q_{0}^{*} \theta$ restricts to $\theta_{-1}^{\#}$ on $T^{-1} \mathcal{G}_{0}^{\#}$. Since equivariancy of $\theta$ follows easily from equivariancy of $\theta_{-1}^{\#}$, this completes the proof.

\subsection{Examples}

It is an easy consequence of the Frobenius theorem that given a contact manifold $M^{\#}$ and a transversal infinitesimal contactomorphism $\xi \in \mathfrak{X}\left(M^{\#}\right)$, there locally exist quotients by $\xi$ for which the fibers are intervals. Hence Theorem 2 shows that locally any transversal infinitesimal automorphism of a parabolic contact structure admits PCS-quotients. Likewise, Theorem 2 shows that any global quotient $q: M^{\#} \rightarrow M$ can be made into a PCS-quotient in case that all fibers of $q$ are simply connected. We now discuss examples which show that in the case of fibers which are circles, the situation is more subtle. Nonetheless, we obtain several interesting examples of global contactifications in which all fibers are circles.

These examples start from the homogeneous models of parabolic contact structures. So we start with a simple Lie group $G$ and a parabolic subgroup $P \subset G$ corresponding to a contact grading of the Lie algebra $\mathfrak{g}$ of $G$, and consider the homogeneous space $G / P$ with the parabolic contact structure coming from the Maurer-Cartan form on $G$. The automorphism group of this geometry is $G$, so looking for quotients, it is natural to consider the actions of 1 parameter subgroups of $G$ on $G / P$. We are particularly interested in finding such actions for which the infinitesimal generator is transversal everywhere, since these may lead to compact quotients.

The first example concerns complex projective space $\mathbb{C} P^{n}$. The Fubini-Study metric on $\mathbb{C} P^{n}$ is a Kähler metric, thus defining a PCS-structure by Proposition 3.3 of [4].

Proposition 1 Consider $S^{2 n+1}$ as the unit sphere in $\mathbb{C}^{n+1}$ and let $q: S^{2 n+1} \rightarrow \mathbb{C} P^{n}$ be the Hopf fibration. Then endowing $S^{2 n+1}$ with its usual CR-structure and $\mathbb{C} P^{n}$ with the PCSstructure of type (PSU $(n+1,1), P)$ defined the Fubini-Study metric, the map $q$ is a global parabolic contactification with circles as fibers.

Proof Recall the description of $S^{2 n+1}$ as the homogeneous model of strictly pseudoconvex CR-structures. Consider $V:=\mathbb{C}^{n+1} \times \mathbb{C}$ endowed with the standard Hermitian form of signature $(n+1,1)$, i.e., the difference of the standard positive definite forms on the two factors. Then any nonzero isotropic vector has to have nonzero first component. Mapping a point $z$ in the unit sphere $S^{2 n+1} \subset \mathbb{C}^{n+1}$ to the line spanned by $(z, 1)$ identifies $S^{2 n+1}$ with the space of isotropic complex lines in $V$. This gives rise to a transitive action of $G:=S U(n+1,1)$ on $S^{2 n+1}$, thus identifying it with $G / P$, where $P$ is the stabilizer of an isotropic line. It is well known that the resulting diffeomorphisms of $S^{2 n+1}$ are exactly those which preserve the strictly pseudoconvex CR-structure induced by the embedding $S^{2 n+1} \hookrightarrow \mathbb{C}^{n+1}$.

Next, the obvious action of $U(1)$ on $S^{2 n+1}$ by complex multiplication can be realized by the action of a 1-parameter subgroup in $S U(n+1,1)$. Namely, for $t \in \mathbb{R}$, one considers multiplication by $e^{i t /(n+2)}$ in the first factor and multiplication by $e^{-i(n+1) t /(n+2)}$ in the second factor. This evidently defines a unitary map of determinant one and the point $\left(e^{i t /(n+2)} z, e^{-i(n+1) t /(n+2)}\right)$ determines the same complex line as $\left(e^{i t} z, 1\right)$. From this it is clear that the infinitesimal generator of this group is simply multiplication by $i$, which maps any point into its real orthocomplement. However, $i z$ never lies in the complex orthocomplement of $z$, which defines the CR subspace. Hence we conclude that the infinitesimal automorphism generating the one-parameter group is transversal everywhere, and by definition the Hopf fibration $q$ is a global space of leaves for this foliation. 
By construction, the $U(1)$-orbits of the action on $S U(n+1,1)$ are $(n+2)$-fold coverings of the $U(1)$ orbits in $S^{2 n+1}$, since the projection sends $\lambda$ to multiplication by $\lambda^{n+2}$. The underlying $G_{0}$-bundle in this case is simply $G / P_{+} \rightarrow G / P$, where $P_{+} \subset P$ is the subgroup introduced in Sect. 2.3. It is well known that its Lie algebra $\mathfrak{p}_{+}$consists of maps vanishing on the distinguished complex line in $\mathbb{C}^{n+2}$, so any element of $P_{+}$acts as the identity on this line. This shows that $P_{+}$intersects our one-parameter subgroup only in the identity, so the projection $G \rightarrow G / P_{+}$restricts to a diffeomorphism on each orbit. Thus Lemma 1 shows that $q: S^{2 n+1} \rightarrow \mathbb{C} P^{n}$ cannot be globally made into a PCS-quotient for the parabolic contact structure of type $(G, P)$.

This is related to the fact that $G$ does not act effectively on $G / P$, and correspondingly $G_{0}$ does not act effectively on $\mathbb{C}^{n}$ (it is an $(n+2)$ )-fold covering of the conformal unitary group $C U(n)$ ). This problem can be resolved by replacing $G$ by the projective group $\underline{G}:=$ $P S U(n+1,1)$ and $P$ by its image $\underline{P}$ in $\underline{G}$. Then $\underline{G} / \underline{P}=G / P$ and now the action becomes effective. Moreover, $\underline{G}$ is the quotient of $G$ by its center, which consists of the $(n+2)$ nd roots of unity times the identity map. This shows that the projection $G \rightarrow G$ restricts to an $(n+2)$-fold covering on $U(1)$-orbits which exactly identifies the different preimages of points in $S^{2 n+1}$. Hence Theorem 2 implies that $q: S^{2 n+1} \rightarrow \mathbb{C} P^{n}$ is globally a PCS-quotient of geometries of type $(\underline{G}, \underline{P})$, for which $\underline{G}_{0}=C U(n)$.

Our second example involves a type of parabolic contact structures which has not been studied intensively in the literature, namely the one associated to the groups $S O^{*}(2 n)$. To formulate the necessary background, consider a finite-dimensional right quaternionic vector space $V$. Then a quaternionically skew Hermitian form is a map $\tau: V \times V \rightarrow \mathbb{H}$, which is bilinear over $\mathbb{R}$ and satisfies $\tau(v, w q)=\tau(v, w) q$ and $\tau(w, v)=-\overline{\tau(v, w)}$ for all $v, w \in V$ and $q \in \mathbb{H}$. It is well known that any finite-dimensional quaternionic vector space admits a unique non-degenerate quaternionically skew Hermitian form up to isomorphism.

The group $S O^{*}(2 n)$ is defined as the group of all quaternionically linear automorphisms of $\mathbb{H}^{n}$ which preserve a non-degenerate quaternionically skew Hermitian form $\tau$. It is easy to see that $\tau$ can be recovered from its real part $\tau_{\mathbb{R}}$, so preserving $\tau$ is equivalent to preserving $\tau_{\mathbb{R}}$. Of course, $\tau_{\mathbb{R}}$ is just a skew symmetric bilinear form $\mathbb{H}^{n} \times \mathbb{H}^{n} \rightarrow \mathbb{R}$ which satisfies $\tau_{\mathbb{R}}(v a, w a)=\tau_{\mathbb{R}}(v, w)$ for any $a=i, j, k$ or equivalently for any unit quaternion $a$.

For $n \geq 3$ consider the quaternionic projective space $\mathbb{H} P^{n}$ of one-dimensional quaternionic subspaces in $\mathbb{H}^{n+1}$. The standard representation of $G L(n+1, \mathbb{H})$ induces a transitive action on $\mathbb{H} P^{n}$, which can be restricted to $G:=S O^{*}(2 n+2)$. However, this subgroup does not act transitively, since the restriction of $\tau$ to a one-dimensional subspace can either be zero or non-degenerate. It is easy to see that $G$ acts transitively on the spaces of non-degenerate lines, which form an open subspace in $\mathbb{H} P^{n}$, and on the space $\mathcal{N}$ of isotropic lines, which is a closed subspace of $\mathbb{H} P^{n}$. This identifies $\mathcal{N}$ with $G / P$, where $P \subset G$ is the stabilizer of an isotropic line in $\mathbb{H}^{n+1}$.

For a quaternionic line $\ell \subset \mathbb{H}^{n+1}$, we can realize $T_{\ell} \mathbb{H} P^{n}$ as $\mathbb{H}^{n+1} / \ell$. Differentiating the defining equation $\tau(v, v)=0$, we see that $\mathcal{N} \subset \mathbb{H} P^{n}$ is a smooth submanifold and that $T_{\ell} \mathcal{N}=\{w \in V: \operatorname{im}(\tau(v, w))=0\} / \ell$, so in particular this submanifold has real codimension three. Moreover, there is a natural subspace $H_{\ell}:=\{w: \tau(v, w)=0\} / \ell \subset$ $T_{\ell} \mathcal{N}$, which has real codimension one. Taking an explicit realization of $G$, it is easy to verify that the codimension-one subbundle $H \subset T \mathcal{N}$ constructed above defines a parabolic contact structure, consisting of a quaternionic structure on $H$ such that the Levi bracket is the real part of a quaternionically skew Hermitian form. One further verifies directly that the resulting subgroup $G_{0}$ is a twofold covering of the group $C S O^{*}(2 n)$ generated by $S O^{*}(2 n)$ and real multiples of the identity. 
On the other hand, consider the complex Grassmannian $\operatorname{Gr}\left(2, \mathbb{C}^{n+1}\right)$ of two planes as a homogeneous space of $S U(n+1)$. Then it is well known that this space admits an invariant complex structure as well as an invariant quaternionic structure, and an invariant Riemannian metric which is Kähler, respectively, quaternion-Kähler with respect to these structures. In Corollary 3.8 of [4] we have shown that the Kähler form of the Kähler metric together with the quaternionic structure defines an $S U(n+1)$-invariant PCS-structure of quaternionic type on $\operatorname{Gr}\left(2, \mathbb{C}^{n+1}\right)$.

Theorem 3 Let $\mathcal{N} \subset \mathbb{H} P^{n}$ be the space of quaternionic lines which are isotropic for a quaternionically skew Hermitian form. Then there is a projection $q: \mathcal{N} \rightarrow \operatorname{Gr}\left(2, \mathbb{C}^{n+1}\right)$, which defines a global parabolic contactification of the $S U(n+1)$-invariant PCS-structure of type $\left(P_{S O}^{*}(2 n), P\right)$ on the Grassmannian with circles as fibers.

Proof Let $\langle$,$\rangle be the standard positive definite quaternionically Hermitian form on V:=$ $\mathbb{H}^{n+1}$. Fix a quaternionically linear map $\mathcal{J}: V \rightarrow V$ such that $\mathcal{J} \circ \mathcal{J}=-$ id and such that $\langle\mathcal{J}(v), \mathcal{J}(w)\rangle=\langle v, w\rangle$. (For example, one can take multiplication by $i$ from the left.) Then it follows immediately that $\tau(v, w):=\langle v, \mathcal{J}(w)\rangle$ is a non-degenerate quaternionically skew Hermitian form, so we can use this form to realize $G:=S O^{*}(2 n+2)$. A quaternionic line $\ell \subset V$ is isotropic for $\tau$ if and only if $\mathcal{J}(\ell) \subset \ell^{\perp}$, the orthocomplement of $\ell$ with respect to $\langle$,$\rangle .$

Scalar multiplication with respect to the complex structure on $V$ defined by $\mathcal{J}$ defines an action of $U$ (1) on $V$ by quaternionically linear maps. From the construction of $\tau$, one readily verifies that this action is orthogonal for $\tau$, so we have found a subgroup of $G$ isomorphic to $U(1)$. By definition, $\tau(v, v)=0$ means that $\langle v, \mathcal{J}(v)\rangle=0$, so $\mathcal{J}(v)$ does not lie in the quaternionic span of $v$. This shows that only $\pm 1 \in U(1)$ acts as the identity on $G / P$. Moreover, for $v \in \ell$ we have $\tau(v, \mathcal{J}(v))=-\langle v, v\rangle \neq 0$, so the infinitesimal automorphism generating this subgroup is transversal on all of $G / P$.

Given an isotropic line $\ell \in \mathcal{N}$, we conclude from $\ell \cap \mathcal{J}(\ell)=\{0\}$ that $\ell \oplus \mathcal{J}(\ell)$ is quaternionic subspace in $V$ of quaternionic dimension two, which in addition is invariant under $\mathcal{J}$. The $U(1)$-orbit of $\ell$ by construction consists of null lines which are contained in this plane. From above, we know that the space of null lines in a quaternionic plane is a codimension three subspace in $\mathbb{H} P^{1} \cong S^{4}$, so it has dimension one, and it is easily verified to be connected. This shows that the $U(1)$-orbit of $\ell$ coincides with the space of those isotropic lines which are contained in $\ell \oplus \mathcal{J}(\ell)$.

Hence, we see that mapping $\ell$ to $\ell \oplus \mathcal{J}(\ell)$ defines a (evidently smooth) map $q$ from $G / P$ to the space of $\mathcal{J}$-invariant quaternionic planes in $V$, whose fibers are exactly the orbits of the $U(1)$-action constructed above. On the other hand, since $\tau(v, \mathcal{J}(v)) \neq 0$ for any $v \in V$, it follows that the restriction of $\tau$ to any $\mathcal{J}$-invariant quaternionic plane $W \subset V$ is non-degenerate. In particular, any such plane contains a family of quaternionic null lines isomorphic to $U(1)$, so $q$ is surjective.

So it remains to show that the space of $\mathcal{J}$-invariant quaternionic planes in $V$ is isomorphic to the complex Grassmannian. Choose two anti-commuting imaginary unit quaternions, for example $i$ and $j$. Then right multiplication by $i$ defines a linear map $V \rightarrow V$ which squares to - id and commutes with $\mathcal{J}$. Hence we obtain a decomposition $V=V^{(1,0)} \oplus V^{(0,1)}$ into two $\mathcal{J}$-invariant summands characterized by $v \cdot i=\mathcal{J}(v)$ for $v \in V^{(1,0)}$ and $v \cdot i=-\mathcal{J}(v)$ for $v \in V^{(0,1)}$. Moreover, one immediately verifies that multiplication by $j$ from the right maps $V^{(1,0)}$ to $V^{(0,1)}$ and vice versa, so both spaces must have complex dimension $n+1$. The projections onto $V^{(1,0)}$ and $V^{(0,1)}$ can be constructed from $\mathcal{J}$ and right multiplication by $i$. A $\mathcal{J}$-invariant quaternionic subspace $W \subset V$ thus is invariant under the projections, and hence $W=\left(W \cap V^{(1,0)}\right) \oplus\left(W \cap V^{(1,0)}\right) \cdot j$. Hence we see that we can identify the space of 
$\mathcal{J}$-invariant quaternionic planes in $V$ with the space of complex planes in $V^{(1,0)}$ and hence with the Grassmannian $\operatorname{Gr}\left(2, \mathbb{C}^{n+1}\right)$.

Hence we see that there is a natural projection $q: \mathcal{N} \rightarrow \operatorname{Gr}\left(2, \mathbb{C}^{n+1}\right)$, which is a quotient by a transversal infinitesimal contactomorphism. The action of $G$ on $\mathcal{N}$ does not descend to the Grassmannian, but one can consider the stabilizer of $\mathcal{J}$ in $G$. It is easy to see that this stabilizer is isomorphic to $U(n+1)$ via the action on $V^{(1,0)}$, and the action on $\operatorname{Gr}\left(2, \mathbb{C}^{n+1}\right)$ further factorizes to $S U(n+1)$. The conformally symplectic structure on the Grassmannian is $S U(n+1)$ invariant and coincides with the one used in Example 3.7 and Corollary 3.8 of [4].

Now as in the first example, the projection $G \rightarrow G / P$ induces a covering on each $U(1)$ orbit but this time, the covering is just twofold. Hence $q$ can be made into a PCS-quotient by passing to the quotient of $G$ by $\mathbb{Z}_{2}=\{ \pm \mathbb{I}\}$, and the resulting PCS-structure of quaternionic type on the Grassmannian is exactly the one from Corollary 3.8 of [4].

\subsection{Contactification of PCS-structures}

For the applications to BGG sequences, we will mainly need the following counterpart to Theorem 2. We show that any local contactification of the conformally symplectic structure underlying a PCS-structure can be canonically made into a PCS-quotient. Together with the result in Lemma 3.1 of [6], this shows that any PCS-structure can be locally realized as a PCS-quotient.

Theorem 4 Suppose that $\left(M^{\#}, H\right)$ is a contact manifold, $(M, \ell)$ is a conformally symplectic manifold and $q: M^{\#} \rightarrow M$ is a reduction by a transverse infinitesimal contactomorphism $\xi \in \mathfrak{X}\left(M^{\#}\right)$.

Then any PCS-structure on $M$, which has $\ell$ as its underlying conformally symplectic structure canonically lifts to a parabolic contact structure on $M^{\#}$ for which $\xi$ is an infinitesimal automorphism.

Proof As discussed in Sect. 2.2, a PCS-structure on $M$ corresponding to the group $G_{0} \subset$ $\operatorname{CSp}\left(\mathfrak{g}_{-1}\right)$ is given by a principal $G_{0}$-bundle $p_{0}: \mathcal{G}_{0} \rightarrow M$ together with a one-form $\theta \in \Omega^{1}\left(\mathcal{G}_{0}, \mathfrak{g}_{-1}\right)$ which is $G_{0}$-equivariant and strictly horizontal. Now we can simply form the pullback $\mathcal{G}_{0}^{\#}:=q^{*} \mathcal{G}_{0} \rightarrow M^{\#}$, which is a principal $G_{0}$-bundle over $M^{\#}$. Explicitly, $\mathcal{G}_{0}^{\#}=\left\{\left(u, x^{\#}\right) \in \mathcal{G}_{0} \times M^{\#}: p(u)=q\left(x^{\#}\right)\right\}$, and the principal right action on this bundle is given by the principal right action of $\mathcal{G}_{0}$ acting on the first factor. Pulling back $\theta$ along the projection onto the first factor, we obtain a smooth $\mathfrak{g}_{-1}$-valued one-form $\theta_{-1}^{\#}$ on $\mathcal{G}_{0}^{\#}$, which evidently is $G_{0}$-equivariant. From the construction it is clear that, for each $x^{\#} \in M^{\#}$, $\theta_{-1}^{\#}\left(u, x^{\#}\right)$ descends to a linear map $T_{x^{\#}} M^{\#} \rightarrow \mathfrak{g}_{-1}$, which restricts to a linear isomorphism on the contact subspace $H_{x^{\#}}$.

Next, there is a unique contact form $\alpha$ on $M^{\#}$ such that $\alpha(\xi) \equiv 1$ and by Proposition 2.2 of [6], there is a unique symplectic form $\omega$ on $M$ which is a section of $\ell$ and satisfies $q^{*} \omega=d \alpha$. By assumption, the conformally symplectic structure induced by the reduction $p_{0}: \mathcal{G}_{0} \rightarrow M$ of structure group is $\ell \subset \Lambda^{2} T^{*} M$. Given a point $u \in \mathcal{G}_{0}$ with $p_{0}(u)=x$, we have $\omega(x) \in \ell_{x}$ so there is a linear isomorphism $\psi(u): \mathfrak{g}_{-2} \rightarrow \mathbb{R}$ such that, viewing $\theta(u)$ as a map $T_{x} M \rightarrow \mathfrak{g}_{-1}$, we get

$$
\omega(x)\left(\eta_{1}, \eta_{2}\right)=\psi(u)\left(\left[\theta(u)\left(\eta_{1}\right), \theta(u)\left(\eta_{2}\right)\right]\right) \quad \forall \eta_{1}, \eta_{2} \in T_{x} M,
$$

where the Lie bracket is in $\mathfrak{g}_{-}$. This defines a smooth map $\psi: \mathcal{G}_{0} \rightarrow L\left(\mathfrak{g}_{-2}, \mathbb{R}\right)$ and equivariancy of $\theta$ implies that $\theta(u \cdot g)(\eta)=\operatorname{Ad}\left(g^{-1}\right)(\theta(u)(\eta))$, and hence $\psi(u \cdot g)=$ $\psi(u) \circ \operatorname{Ad}(g)$. 
Using this, we now define $\theta_{-2}^{\#} \in \Omega^{1}\left(\mathcal{G}_{0}^{\#}, \mathfrak{g}_{-2}\right)$ by $\theta_{-2}^{\#}\left(u, x^{\#}\right)=\psi(u)^{-1} \circ\left(p_{0}^{\#}\right)^{*} \alpha$, where $p_{0}^{\#}: \mathcal{G}_{0}^{\#} \rightarrow M^{\#}$ is the canonical projection. This evidently is a smooth one-form, and since $\left(p_{0}^{\#}\right)^{*} \alpha$ is invariant under the principal right action, we see that $\theta_{-2}^{\#}$ is $G_{0}$-equivariant. Next, the kernel of $\theta_{-2}^{\#}$ in a point coincides with the kernel of $\left(p_{0}^{\#}\right)^{*} \alpha$ in that point and thus with the preimage $\left(T p_{0}^{\#}\right)^{-1}(H)$. Finally, if $\tilde{\eta}_{1}$ and $\tilde{\eta}_{2}$ are two sections of this subbundle, then we compute

$$
\begin{aligned}
d \theta_{-2}^{\#}\left(u, x^{\#}\right)\left(\tilde{\eta}_{1}, \tilde{\eta}_{2}\right) & =-\theta_{-2}^{\#}\left(u, x^{\#}\right)\left(\left[\tilde{\eta}_{1}, \tilde{\eta}_{2}\right]\right)=-\psi(u)^{-1}\left(\left(p_{0}^{\#}\right)^{*} \alpha\left(u, x^{\#}\right)\left(\left[\tilde{\eta}_{1}, \tilde{\eta}_{2}\right]\right)\right. \\
& =\psi(u)^{-1}\left(d\left(p_{0}^{\#}\right)^{*} \alpha\left(u, x^{\#}\right)\left(\tilde{\eta}_{1}, \tilde{\eta}_{2}\right)\right) .
\end{aligned}
$$

Now since $d\left(p_{0}^{\#}\right)^{*} \alpha=\left(p_{0}^{\#}\right)^{*} d \alpha=\left(p_{0}^{\#}\right)^{*} q^{*} \omega$, we get, putting $x=q\left(x^{\#}\right)$ and denoting by $\eta_{i} \in T_{x} M$ the value of $\tilde{\eta}_{i}\left(u, x^{\#}\right)$ under the natural projection for $i=1,2$ :

$$
\begin{aligned}
d \theta_{-2}^{\#}\left(u, x^{\#}\right)\left(\tilde{\eta}_{1}, \tilde{\eta}_{2}\right) & =\psi(u)^{-1}\left(\omega(x)\left(\eta_{1}, \eta_{2}\right)\right)=\left[\theta(u)\left(\eta_{1}\right), \theta(u)\left(\eta_{2}\right)\right] \\
& =\left[\theta_{-1}^{\#}\left(u, x^{\#}\right)\left(\tilde{\eta}_{1}\right), \theta_{-1}^{\#}\left(u, x^{\#}\right)\left(\tilde{\eta}_{2}\right)\right],
\end{aligned}
$$

where the Lie bracket is in $\mathfrak{g}_{-}$. Together with the above properties, this shows that $\theta^{\#}:=$ $\left(\theta_{-2}^{\#}, \theta_{-1}^{\#}\right)$ makes $p_{0}^{\#}: \mathcal{G}_{0}^{\#} \rightarrow M^{\#}$ into a regular infinitesimal flag structure on $M^{\#}$, see Sect. 2.3. Since $\mathfrak{g}$ is not of type $C_{n}$, this is equivalent to a parabolic contact structure of type $(G, P)$.

So it remains to show that $\xi$ is an infinitesimal automorphism of this parabolic contact structure or equivalently of the infinitesimal flag structure. Observe first that $T_{\left(u, x^{\#}\right)} \mathcal{G}_{0}^{\#}=$ $\left\{\left(\eta_{1}, \eta_{2}\right) \in T_{u} \mathcal{G}_{0} \times T_{x^{\#}} M^{\#}: T_{u} p \cdot \eta_{1}=T_{x^{\#}} q \cdot \eta_{2}\right\}$, and hence $\tilde{\xi}:=(0, \xi)$ is a well-defined vector field on $\mathcal{G}_{0}^{\#}$. The flow of this vector field is clearly given by $\mathrm{Fl}_{t}^{\tilde{\xi}}\left(u, x^{\#}\right)=\left(u, \mathrm{Fl}_{t}^{\xi}\left(x^{\#}\right)\right)$ and it suffices to prove that this flow preserves $\theta^{\#}$.

Take a point $\left(u, x^{\#}\right) \in \mathcal{G}_{0}^{\#}$ and a tangent vector $\left(\eta_{1}, \eta_{2}\right) \in T_{\left(u, x^{\#}\right)} \mathcal{G}_{0}^{\#}$. Then

$$
T_{\left(u, x^{\#}\right)} \mathrm{Fl}_{t}^{\tilde{\xi}} \cdot\left(\eta_{1}, \eta_{2}\right)=\left(\eta_{1}, T_{x^{\#}} \mathrm{Fl}_{t}^{\xi} \cdot \eta_{2}\right),
$$

wherever the right-hand side is defined. But by definition

$$
\theta_{-1}^{\#}\left(u, x^{\#}\right)\left(\eta_{1}, \eta_{2}\right)=\theta(u)\left(\eta_{1}\right),
$$

which immediately implies $\left(\mathrm{Fl}_{t}^{\tilde{\xi}}\right)^{*} \theta_{-1}^{\#}=\theta_{-1}^{\#}$, whenever the flow is defined. Again by definition, we get

$$
\theta_{-2}^{\#}\left(u, x^{\#}\right)\left(\eta_{1}, \eta_{2}\right)=\psi(u)^{-1}\left(\alpha\left(x^{\#}\right)\left(\eta_{2}\right)\right),
$$

and hence $\left(\mathrm{Fl}_{t}^{\tilde{\xi}}\right)^{*} \theta_{-2}^{\#}=\theta_{-2}^{\#}$ follows immediately from $\left(\mathrm{Fl}_{t}^{\xi}\right)^{*} \alpha=\alpha$.

\subsection{Local uniqueness of PCS-contactifications}

To complete the picture, we have to prove that different realizations of PCS-structures as PCS-quotients as constructed in Theorem 4 are locally compatible. The corresponding result for conformally symplectic structures has been proved in Proposition 3.1 of [6], so we only have to prove compatibility with the additional structures.

Theorem 5 Suppose that $M$ and $\tilde{M}$ carry a PCS-structure of some fixed type with underlying conformally symplectic structures $\ell$ and $\tilde{\ell}$. Suppose further that $\left(M^{\#}, H\right)$ and $\left(\tilde{M}^{\#}, \tilde{H}\right)$ are contact manifolds and that $q: M^{\#} \rightarrow M$ and $\tilde{q}: \tilde{M}^{\#} \rightarrow \tilde{M}$ are reductions with respect to 
transverse symmetries $\xi \in \mathfrak{X}\left(M^{\#}\right)$ and $\tilde{\xi} \in \mathfrak{X}\left(\tilde{M}^{\#}\right)$. Suppose finally that $\varphi: M \rightarrow \tilde{M}$ is a PCS-diffeomorphism and that $\varphi^{\#}: M^{\#} \rightarrow \tilde{M}^{\#}$ is a contactomorphism which lifts $\varphi$.

Then $\left(\varphi^{\#}\right)^{*} \tilde{\xi}=\lambda \xi$ for a nowhere vanishing, locally constant function $\lambda$ and $\varphi^{\#}$ is an automorphism of the lifted parabolic contact structures from Theorem 4.

Proof Since $\varphi^{\#}$ is a contactomorphism, $\left(\varphi^{\#}\right)^{*} \tilde{\xi} \in \mathfrak{X}\left(M^{\#}\right)$ is a transverse infinitesimal automorphism of the contact structure $H \subset T M^{\#}$. On the other hand, since $\varphi^{\#}$ lifts $\varphi$ it has to map fibers of $q$ to fibers of $\tilde{q}$, which shows that $\left(\varphi^{\#}\right)^{*} \tilde{\xi}=\lambda \xi$ for some smooth function $\lambda$ on $M^{\#}$. But then for $\eta \in \mathfrak{X}\left(M^{\#}\right)$ we get $[\lambda \xi, \eta]=\lambda[\xi, \eta]-(\eta \cdot \lambda) \xi$, and if $\eta \in \Gamma(H)$, then by assumption both the left-hand side and the first summand in the right-hand side are sections of $H$. Since $\xi$ is transverse, this implies that $\eta \cdot \lambda=0$ for any $\eta \in \Gamma(H)$ and since $H$ is bracket-generating this shows that $\lambda$ is locally constant.

Now let $\left(p: \mathcal{G}_{0} \rightarrow M, \theta\right)$ and $\left(\tilde{p}: \tilde{\mathcal{G}}_{0} \rightarrow \tilde{M}, \tilde{\theta}\right)$ be the bundles defining the PCSstructures. Then by assumption there is an isomorphism $\Phi: \mathcal{G}_{0} \rightarrow \tilde{\mathcal{G}}_{0}$ of principal $G_{0}-$ bundles such that $\tilde{p} \circ \Phi=\varphi \circ p$ and such that $\Phi^{*} \tilde{\theta}=\theta$. Forming the pullback bundles as in the proof of Theorem 4, we see that

$$
\left(u, x^{\#}\right) \mapsto\left(\Phi(u), \varphi^{\#}\left(x^{\#}\right)\right)
$$

defines an isomorphism $\left(\Phi, \varphi^{\#}\right): \mathcal{G}_{0}^{\#} \rightarrow \tilde{\mathcal{G}}_{0}^{\#}$ lifting $\varphi^{\#}$, so it remains to prove that this is compatible with the frame forms on the two bundles constructed in the proof of Theorem 4 . Inserting the definitions, one immediately verifies that

$$
\left(\Phi, \varphi^{\#}\right)^{*} \tilde{\theta}_{-1}^{\#}\left(u, x^{\#}\right)\left(\eta_{1}, \eta_{2}\right)=\Phi^{*} \tilde{\theta}(u)\left(\eta_{1}\right)=\theta(u)\left(\eta_{1}\right),
$$

and thus $\left(\Phi, \varphi^{\#}\right)^{*} \tilde{\theta}_{-1}^{\#}=\theta_{-1}^{\#}$. On the other hand, since $\left(\varphi^{\#}\right)^{*} \tilde{\xi}=\lambda \xi$, we see that the contact forms corresponding to $\xi$ and $\tilde{\xi}$ are related by $\left(\varphi^{\#}\right)^{*} \tilde{\alpha}=\frac{1}{\lambda} \alpha$. Since $\frac{1}{\lambda}$ is locally constant, the corresponding symplectic forms are related by $\varphi^{*} \tilde{\omega}=\frac{1}{\lambda} \omega$. Viewing the values of $\theta$ and $\tilde{\theta}$ as linear isomorphisms on tangent spaces of $M$ and $\tilde{M}$, the fact that $\Phi^{*} \tilde{\theta}=\theta$ reads as

$$
\tilde{\theta}(\Phi(u))\left(T_{p(u)} \varphi \cdot \eta\right)=\theta(u)(\eta)
$$

for all $u \in \mathcal{G}_{0}$ and $\eta \in T_{p(u)} M$. Together with $\varphi^{*} \tilde{\omega}=\frac{1}{\lambda} \omega$, this implies that the isomorphisms $\psi$ and $\tilde{\psi}$ as constructed in the proof of Theorem 4 satisfy $\tilde{\psi}(\Phi(u))=\frac{1}{\lambda} \psi(u)$. Using this, we compute

$$
\begin{aligned}
\left(\Phi, \varphi^{\#}\right)^{*} \tilde{\theta}_{-2}^{\#}\left(u, x^{\#}\right)\left(\eta_{1}, \eta_{2}\right) & =\tilde{\theta}_{-2}^{\#}\left(\Phi(u), \varphi^{\#}\left(x^{\#}\right)\right)\left(T_{u} \Phi \cdot \eta_{1}, T_{x^{\#}} \varphi^{\#} \cdot \eta_{2}\right) \\
& =\tilde{\psi}(\Phi(u))^{-1}\left(\left(\varphi^{\#}\right)^{*} \tilde{\alpha}\left(x^{\#}\right)\left(\eta_{2}\right)\right)=\lambda \psi(u)^{-1}\left(\frac{1}{\lambda} \alpha\left(x^{\#}\right)\left(\eta_{2}\right)\right),
\end{aligned}
$$

so $\left(\Phi, \varphi^{\#}\right)^{*} \tilde{\theta}_{-2}^{\#}=\theta_{-2}^{\#}$.

\subsection{Distinguished connections and contactifications}

As shown in [4], any PACS-structure on $M$ determines a canonical compatible linear connection on $T M$. In the case of PCS-structures, these are closely related to special symplectic connections as studied in [3], see Section 4.7 of [4] for a discussion of the relation. We now conclude the discussion of parabolic contactifications by relating the distinguished connection associated to a PCS-quotient of a parabolic contact structure to distinguished connections "upstairs". On the one hand, this provides alternative proofs for and extensions of some results from Cahen and Schwachhöfer [3], on the other hand, the result will be useful for the discussion of conformally Fedosov structures in Sect. 3 below as well as for the third part of this series. 
A family of distinguished connections associated to a parabolic contact structure is obtained via so-called Weyl structures as discussed in Chapter 5 of [7]. Consider the Cartan geometry $\left(\mathcal{G}^{\#} \rightarrow M^{\#}, \omega\right)$ of type $(G, P)$ determined by a parabolic contact structure and the underlying bundle $\mathcal{G}_{0}^{\#} \rightarrow M$. A Weyl structure is defined as a $G_{0}$-equivariant section $\sigma$ of the natural projection $\mathcal{G}^{\#} \rightarrow \mathcal{G}_{0}^{\#}$. Taking the component $\omega_{0} \in \Omega^{1}\left(\mathcal{G}^{\#}, \mathfrak{g}_{0}\right)$ of the Cartan connection in $\mathfrak{g}_{0} \subset \mathfrak{g}$, the pull back $\sigma^{*} \omega_{0}$ is a principal connection on $\mathcal{G}_{0}^{\#}$ called the Weyl connection determined by $\sigma$.

A principal connection on $\mathcal{G}_{0}^{\#}$ can be equivalently viewed as a linear connection $\nabla^{\#}$ on the vector bundle $H \rightarrow M^{\#}$, which is compatible with the reduction of structure group defined by $\mathcal{G}_{0}^{\#}$. In particular, $\nabla^{\#}$ is a contact connection, in the sense that the induced connection on $\Lambda^{2} H$ preserves the subbundle $\Lambda_{0}^{2} H$ formed by the kernel of the Levi bracket.

Further, the pull back of the components in $\mathfrak{g}_{-2} \oplus \mathfrak{g}_{-1}$ of the Cartan connection $\omega$ along $\sigma$ can be interpreted as defining an isomorphism between $T M^{\#}$ and its associated graded vector bundle $H \oplus Q$, where $Q:=T M^{\#} / H$. In particular, this defines a projection $\pi$ from $T M^{\#}$ onto the subbundle $H \subset T M^{\#}$. Using this projection, we can define the contact torsion of $\nabla^{\#}$ via

$$
T\left(\eta_{1}, \eta_{2}\right)=\nabla_{\eta_{1}}^{\#} \eta_{2}-\nabla_{\eta_{2}}^{\#} \eta_{1}-\pi\left(\left[\eta_{1}, \eta_{2}\right]\right)
$$

for sections $\eta_{1}, \eta_{2} \in \Gamma(H)$. This expression is immediately seen to be bilinear over smooth functions and thus defines a bundle map $T: \Lambda^{2} H \rightarrow H$.

We have to recall a few further facts on Weyl structures from Section 5.2.11 of [7]. The line bundle $Q$ can be used as a so-called bundle of scales, so passing to the induced linear connection on $Q$ defines a bijection between Weyl structures and linear connections on $Q$. Now consider a reduction $q: M^{\#} \rightarrow M$ by a transverse symmetry $\xi \in \mathfrak{X}\left(M^{\#}\right)$. Then we can project $\xi$ to a section of $Q$, which by construction is nowhere vanishing. Of course, there is a unique linear connection on $Q$, for which this section is parallel, and this in turn uniquely determines a Weyl structure $\sigma$. This will be called the Weyl structure associated to $\xi$.

Theorem 6 Let $q_{0}: \mathcal{G}_{0}^{\#} \rightarrow \mathcal{G}_{0}$ be a PCS-quotient with respect to a transverse infinitesimal automorphism $\xi_{0} \in \mathfrak{X}\left(\mathcal{G}_{0}^{\#}\right)$ with base map $q: M^{\#} \rightarrow M$.

Then there is a unique principal connection form $\gamma^{\#}$ on $p_{0}^{\#}: \mathcal{G}_{0}^{\#} \rightarrow M^{\#}$ for which $\xi_{0} \in \mathfrak{X}\left(\mathcal{G}_{0}^{\#}\right)$ is horizontal and which coincides with the principal connection form determined by the Weyl structure associated to $\xi$ on $T^{-1} \mathcal{G}_{0}^{\#} \subset T \mathcal{G}_{0}^{\#}$. The form $\gamma^{\#}$ descends to a principal connection form on $\mathcal{G}_{0} \rightarrow M$, which corresponds to the canonical connection associated to the PCS-structure from Theorem 1.

Proof Let $\sigma: \mathcal{G}_{0}^{\#} \rightarrow \mathcal{G}^{\#}$ be the Weyl structure determined by $\xi$ and let $\tilde{\gamma}^{\#}=\sigma^{*} \omega_{0}$ be the corresponding Weyl connection. Since $\xi_{0}$ is $G_{0}$-invariant, the function $\tilde{\gamma}^{\#}\left(\xi_{0}\right): \mathcal{G}_{0}^{\#} \rightarrow \mathfrak{g}_{0}$ is $G_{0}$-equivariant. Next, let $\alpha \in \Omega^{1}\left(M^{\#}\right)$ be the unique contact form with $\alpha(\xi)=1$ and put $\gamma^{\#}:=\tilde{\gamma}^{\#}-\tilde{\gamma}^{\#}\left(\xi_{0}\right)\left(p_{0}^{\#}\right)^{*} \alpha$. This is evidently $G_{0}$-equivariant, and since $\left(p_{0}^{\#}\right)^{*} \alpha$ vanishes on $T^{-1} \mathcal{G}_{0}^{\#}$, it is a principal connection form which coincides with $\tilde{\gamma}^{\#}$ on $T^{-1} \mathcal{G}_{0}^{\#}$. Since $\xi_{0}$ lifts $\xi$, we get $\gamma^{\#}\left(\xi_{0}\right)=0$, so we have verified the required properties. Of course, these pin down $\gamma^{\#}$ uniquely.

By definition, the fibers of $q_{0}: \mathcal{G}_{0}^{\#} \rightarrow \mathcal{G}_{0}$ are connected and can locally be represented as flow lines of $\xi_{0}$. Hence by Corollary 2.3 in [2] we can prove that $\gamma^{\#}$ descends to $\mathcal{G}_{0}$ by showing that it is invariant under the flow of $\xi_{0}$. As discussed in Sect. 2.3, the infinitesimal automorphism $\xi_{0}$ uniquely lifts to a $P$-invariant vector field $\tilde{\xi} \in \mathfrak{X}(\mathcal{G})$ whose local flows preserve the Cartan connection $\omega$. Supposing that both flows are defined, the map $\mathrm{Fl}_{-t}^{\tilde{\xi}} \circ \tilde{\sigma} \circ$ $\mathrm{Fl}_{t}^{\xi_{0}}$ is again a Weyl structure. Since $\left(\mathrm{Fl}_{t}^{\tilde{\xi}}\right)^{*} \omega=\omega$, we conclude that $\left(\mathrm{Fl}_{t}^{\xi_{0}}\right)^{*} \tilde{\gamma}^{\#}$ is the Weyl 
connection associated to this pulled back Weyl structure. Thus we can prove invariance of $\tilde{\gamma}^{\#}$ under the flow of $\xi_{0}$ by showing that $\mathrm{Fl}_{-t}^{\tilde{\xi}} \circ \tilde{\sigma} \circ \mathrm{Fl}_{t}^{\xi_{0}}=\sigma$ for small $t$.

Denoting by $\tau \in \Gamma(Q)$ the section obtained by projecting $\xi$, it is evident that $\left(\mathrm{Fl}_{t}^{\xi}\right)^{*} \tau$ is parallel for the Weyl connection corresponding to $\mathrm{Fl}_{-t}^{\tilde{\xi}} \circ \tilde{\sigma} \circ \mathrm{Fl}_{t}^{\xi_{0}}$, and we know from above that this characterizes the pulled back Weyl structure. But since $\tau$ is a projection of $\xi$ and the Lie derivative of $\xi$ along $\xi$ equals $[\xi, \xi]=0$, we conclude $\left(\mathrm{Fl}_{t}^{\xi}\right)^{*} \tau=\tau$, so $\mathrm{Fl}_{-t}^{\tilde{\xi}} \circ \tilde{\sigma} \circ \mathrm{Fl}_{t}^{\xi_{0}}=\sigma$.

Knowing that $\tilde{\gamma}^{\#}$ is invariant under the flow of $\xi_{0}$, it follows readily that the same is true for the function $\tilde{\gamma}^{\#}\left(\xi_{0}\right)$. Also, the pullback of $\alpha$ clearly is invariant under the flow of $\xi_{0}$, since $\alpha$ is invariant under the flow of $\xi$. Thus we conclude that $\gamma^{\#}$ is invariant under the flow of $\xi_{0}$ and hence descends to a $\mathfrak{g}_{0}$-valued one-form on $\mathcal{G}_{0}$, which then clearly is a principal connection form on $\mathcal{G}_{0} \rightarrow M$.

To complete the proof it thus suffices to prove that the corresponding linear connection on $T M$ has algebraically harmonic torsion. Now the torsion of a linear connection on $T M$ can be computed from the corresponding principal connection by evaluating the exterior derivative of the soldering form on horizontal lifts of the vector fields. Now the soldering form on $\mathcal{G}_{0}$ pulls back to the form $\theta_{-1}^{\#}$ on $\mathcal{G}_{0}^{\#}$. This can be extended to a one-form on $\mathcal{G}_{0}^{\#}$ by requiring that it vanishes on $\xi_{0}$ (which corresponds to the isomorphism between $T M^{\#}$ and $H \oplus Q$ defined by the Weyl structure determined by $\xi$ ). Evaluating the exterior derivative of this one-form on the horizontal lifts sections of $H \rightarrow M^{\#}$, one obtains the function corresponding to the contact torsion of the corresponding Weyl connection. This contact torsion is well known to have values in $\operatorname{ker}(\square)$, see Theorem 5.2.11 of [7].

Now consider the definition of the contact torsion $T\left(\eta_{1}, \eta_{2}\right)$ in case that $\eta_{1}$ and $\eta_{2}$ are local lifts of vector fields on $M$. Then $\left[\eta_{1}, \eta_{2}\right]$ is a local lift of their Lie bracket and this differs from $\pi\left(\left[\eta_{1}, \eta_{2}\right]\right)$ by a multiple of $\xi$. Thus $\pi\left(\left[\eta_{1}, \eta_{2}\right]\right)$ is the section of $H$ lifting the Lie bracket of the downstairs vector fields. This immediately implies that this contact torsion coincides with the torsion of the linear connection associated to $\gamma$, which completes the proof.

\subsection{Contactifications and special symplectic connections}

Using Theorem 6, we can now complete the discussion of the relation between PCS-structures and special symplectic connections in the sense of Cahen and Schwachhöfer [3]. Consider a contact grading of a simple Lie algebra $\mathfrak{g}$, which is not of type $C_{n}$, and let $\mathfrak{g}_{0} \subset \mathfrak{c s p}\left(\mathfrak{g}_{-1}\right)$ be the corresponding reductive subalgebra. As discussed in Section 4.7 of [4], the intersection $\mathfrak{g}_{0}^{0}$ of $\mathfrak{g}_{0}$ with $\mathfrak{s p}\left(\mathfrak{g}_{-1}\right)$ is called a special symplectic subalgebra and there is a corresponding concept of special symplectic connections. In particular, that class contains all linear connections with exceptional holonomy that preserve a symplectic form.

In Theorem 4.7 of [4], we have shown that any special symplectic connection of type $\mathfrak{g}$ is the distinguished connection of some torsion-free PCS-structure of type $(G, P)$ for a group $G$ with Lie algebra $\mathfrak{g}$. Now we can characterize the special symplectic connections among these distinguished connections via local flatness of contactifications.

Theorem 7 Consider a PCS-structure of type $(G, P)$ on a smooth manifold $M$ and let $\nabla$ be the distinguished connection on $T M$ determined by this structure. Then $\nabla$ is a special symplectic connection if and only if any local parabolic contactification of $M$ is locally flat (as a parabolic contact structure).

Proof For a local parabolic contactification $M^{\#}$ of $M$ with corresponding infinitesimal automorphism $\xi$, we have seen in the last part of the proof of Theorem 6 that the contact torsion of the Weyl connection $\nabla^{\#}$ determined by $\xi$ coincides with the torsion of $\nabla$. Torsion freeness 
of $\nabla$ thus implies vanishing of the contact torsion of $\nabla^{\#}$. But by Lemma 4.2.2 of [7], all the harmonic torsion of a parabolic contact structure is contained in the contact torsion, so torsion freeness of $\nabla$ is equivalent to vanishing of the harmonic torsion of the parabolic geometry on $M^{\#}$.

From the discussion of parabolic contact structures in Section 4.2 of [7] it follows that for $\mathfrak{g}$ not of type $A_{n}$, vanishing of the harmonic torsion is equivalent to local flatness of the parabolic geometry. On the other hand, by Theorem 4.7 of [4], for $\mathfrak{g}$ not of type $A_{n}$, torsion freeness of $\nabla$ is equivalent to $\nabla$ being special symplectic, so the proof is complete in this case.

For $\mathfrak{g}$ of type $A_{n}$, Proposition 4.2.3 and the discussion in Section 4.2.4 in [7] show that vanishing of the harmonic torsion of the parabolic geometry on $M^{\#}$ is equivalent to torsion freeness of this geometry. Moreover, we can apply part (3) of Theorem 4.2.2 of [7] to $\nabla^{\#}$ and conclude that the harmonic curvature component in homogeneity two of this geometry (which is the only remaining component in dimensions $\geq 5$ ) can be recovered as a component of the curvature of $\nabla^{\#}$. This curvature is a two-form with values in $\operatorname{End}(H)$ and one has to restrict this two-form to $\Lambda_{0}^{2} H^{*} \subset \Lambda^{2} T^{*} M$, the kernel of the Levi bracket within $\Lambda^{2} H$. This defines a section of the bundle associated to $\Lambda_{0}^{2} \mathfrak{g}_{1} \otimes \mathfrak{g}_{0}$, and one has to further restrict to the kernel of the Kostant-Laplacian $\square$ on this representation. But on $\Lambda_{0}^{2} H^{*}$, one can also compute the curvature via

$$
R\left(\eta_{1}, \eta_{2}\right)\left(\eta_{3}\right)=\nabla_{\eta_{1}}^{\#} \nabla_{\eta_{2}}^{\#} \eta_{3}-\nabla_{\eta_{2}}^{\#} \nabla_{\eta_{1}}^{\#} \eta_{3}-\nabla_{\pi\left(\left[\eta_{1}, \eta_{2}\right]\right)}^{\#} \eta_{3}
$$

This shows that the relevant component of the curvature of $\nabla^{\#}$ is just the pullback of the curvature of $\nabla$. Hence we see that the parabolic contact structure on $M^{\#}$ is locally flat if and only if the component of its curvature in the subbundle corresponding to $\operatorname{ker}(\square) \subset \Lambda^{2} \mathfrak{g}_{1} \otimes \mathfrak{g}_{0}$ vanishes identically. This subspace is well known to be the $\mathfrak{g}_{0}$-irreducible component of maximal highest weight, which immediately implies that this condition is equivalent to vanishing of the Bochner curvature of $\nabla$ and thus the result.

\section{Conformally Fedosov structures and their contactifications}

In the previous discussions, we have excluded type $C_{n}$, since in this case $\mathfrak{g}_{0}=\mathfrak{c} \mathfrak{s p}\left(\mathfrak{g}_{-1}\right)$. This corresponds to the fact that the parabolic contact structure determined by this grading is not defined by a regular infinitesimal flag structure in the sense of Sect. 2.3. The contact grading of $\mathfrak{s p}(2 n, \mathbb{R})$ gives rise to so-called contact projective structures, an analog of classical projective structures. In this section, we discuss a geometric structure refining a conformally symplectic structure, which can be thought of as the PCS-structure associated to the contact grading of $\mathfrak{s p}(2 n, \mathbb{R})$. (It seems to be possible to define a similar concept related to almost conformally symplectic structures, but since contactifications are only available in the conformally symplectic case, we do not pursue this direction here.)

Initially, this structure can be described as a projective structure compatible with a conformally symplectic structure. However, there is a counterpart to the vanishing of the first prolongation for PACS-structures, which implies that such a geometry is given by a conformally symplectic structure and a torsion-free affine connection preserving this structure. Locally, such a structure is even equivalent to a symplectic structure together with a torsionfree symplectic connection. These geometries were originally introduced by Eastwood and Slovák (in a slightly different presentation) in the first version of [8] under the name "conformally Fedosov structures", and we will keep this name here. 


\subsection{Projective structures and conformally Fedosov structures}

Recall that two torsion-free linear connections $\nabla$ and $\hat{\nabla}$ on the tangent bundle of a smooth manifold $M$ are called projectively equivalent if and only if they have the same geodesics up to parametrization. This can be equivalently characterized as the existence of a one-form $\Upsilon$ on $M$ such that

$$
\hat{\nabla}_{\xi \eta}=\nabla_{\xi} \eta+\Upsilon(\xi) \eta+\Upsilon(\eta) \xi,
$$

for all $\xi, \eta \in \mathfrak{X}(M)$. A projective structure on a smooth manifold $M$ is then defined as a projective equivalence class of torsion-free connections on $M$. Equivalently, it can be viewed as being given by the family of one-dimensional submanifolds formed by the geodesic paths of the connections in the class.

Definition 2 Let $\left(M, \ell \subset \Lambda^{2} T^{*} M\right)$ be a conformally symplectic manifold. A conformally Fedosov structure on $(M, \ell)$ is a projective structure $[\nabla]$, which contains a connection that preserves $\ell$.

The following characterization is essentially contained in [8], we reproduce it for convenience of the reader. We use abstract index notation as in [8].

Proposition 2 For a projective equivalence class $\Phi$ of torsion-free affine connections on a conformally symplectic manifold $(M, \ell)$ of dimension $\geq 4$, the following conditions are equivalent:

(i) $\Phi$ defines a conformally Fedosov structure on $(M, \ell)$.

(ii) For one (or equivalently any) connection $\nabla=\nabla_{a}$ in the class $\Phi$ and one non-vanishing (or equivalently any) local section $\omega=\omega_{b c}$ of $\ell$, we have $\nabla_{(a} \omega_{b) c}=\varphi_{(a} \omega_{b) c}$ for some one-form $\varphi=\varphi_{a}$.

(iii) There is a unique connection $\nabla_{a}$ in $\Phi$ which preserves $\ell$.

Moreover, the connection on $\ell$ induced by the connection $\nabla$ from (iii) is flat with any local closed section being parallel. In particular, locally $\nabla$ is a torsion-free symplectic connection with respect to each of these closed sections.

Proof The formula for the change of the induced connection on two-forms from [8] says that changing from $\nabla_{a}$ to $\hat{\nabla}_{a}$ corresponding to a one-form $\Upsilon_{a}$ one gets

$$
\hat{\nabla}_{a} \omega_{b c}=\nabla_{a} \omega_{b c}-2 \Upsilon_{a} \omega_{b c}-\Upsilon_{b} \omega_{a c}-\Upsilon_{c} \omega_{b a} .
$$

If we symmetrize over $(a, b)$, then the last term does not contribute and we get

$$
\hat{\nabla}_{(a} \omega_{b) c}=\nabla_{(a} \omega_{b) c}-3 \Upsilon_{(a} \omega_{b) c} .
$$

This shows that if the condition in (ii) is satisfied for one connection in the projective class $\Phi$, then it is satisfied for all such connections. Likewise, one easily verifies that if the condition in (ii) is satisfied for one non-vanishing section, then it is satisfied for any section of $\ell$.

Condition (i) by definition means that there is a connection $\nabla_{a}$ in $\Phi$ such that for any local section $\omega_{b c}$ of $\ell$, we have $\nabla_{a} \omega_{b c}=\varphi_{a} \omega_{b c}$ for some one-form $\varphi=\varphi_{a}$. Thus (i) implies (ii) and evidently (iii) implies (i), so we can complete the proof by showing that (ii) implies (iii).

To do this, take a local non-vanishing section $\omega_{b c}$ of $\ell$, which is closed as a two-form on $M$, and any connection $\nabla_{a}$ in the class $\Phi$. Then by (ii) there is a one-form $\varphi_{a}$ such that $\nabla_{(a} \omega_{b) c}=\varphi_{(a} \omega_{b) c}$. Passing to the projectively equivalent connection $\hat{\nabla}$ corresponding to 
$\Upsilon_{a}=\frac{1}{3} \varphi_{a}$ we see from above that $\hat{\nabla}_{(a} \omega_{b) c}=0$. This means that apart from the apparent skew symmetry in $b$ and $c, \hat{\nabla}_{a} \omega_{b c}$ is also skew symmetric in $a$ and $b$. But this easily implies that the complete alternation of $\hat{\nabla}_{a} \omega_{b c}$ is a multiple of $\hat{\nabla}_{a} \omega_{b c}$, and since this alternation coincides with $d \omega=0$, we see that $\omega$ is parallel for $\hat{\nabla}$. This implies the existence part of (iii) as well as the last claim.

To prove the uniqueness part of (iii), observe that by part (4) of Proposition 2.3 of [4] every torsion-free connection compatible with $\ell$ has the property that any local closed section of $\ell$ is parallel for the induced connection. But if $\omega$ is any such section, then by non-degeneracy $\omega^{n}=\omega \wedge \cdots \wedge \omega$ is a volume form on $M$, which clearly is parallel for the induced connection. But it is a well-known fact in the theory of projective structures that a projective class can contain at most one connection which leaves some fixed volume form parallel.

\subsection{Contact projective structures}

A parabolic contact structure corresponding to the contact grading of a simple Lie algebra of type $C_{n}$ is a so-called contact projective structure. As discussed above, this geometry is exceptional among parabolic contact structures, since it is not equivalent to an underlying regular infinitesimal flag structure. Rather than that, it can be described in terms of an equivalence class of compatible (partial) connections.

To recall the necessary notions, let $M^{\#}$ be a smooth manifold endowed with a contact structure $H \subset T M^{\#}$. A partial connection on $H$ is a bilinear operator $\nabla: \Gamma(H) \times \Gamma(H) \rightarrow$ $\Gamma(H)$, which is linear over smooth functions in the first variable and satisfies the usual Leibniz rule in the second variable. So this is like a covariant derivative, but it is possible to differentiate only in directions lying in the contact subbundle. It is no problem to form the induced partial connection on $\Lambda^{2} H$, and parallel to Sect. 2.9 a partial contact connection is one that preserves $\Lambda_{0}^{2} H$. Since the Levi bracket identifies $\Lambda^{2} H / \Lambda_{0}^{2} H$ with $Q=T M^{\#} / H$, we get an induced partial connection $\nabla^{Q}$ on the line bundle $Q$.

Using this, one can next associate a projection $\pi: T M^{\#} \rightarrow H$ to a partial contact connection $\nabla$, see Sections 4.2.2 and 4.2.6 of [7]. This is uniquely characterized by the fact that for $\zeta \in \mathfrak{X}\left(M^{\#}\right)$ and $\eta \in \Gamma(H)$ we have

$$
\mathcal{L}(\pi(\zeta), \eta)=\nabla_{\eta}^{Q}(\zeta+H)-([\eta, \zeta]+H)
$$

here $\zeta+H$ denotes the section of $Q$ determined by $\zeta$ and likewise for the Lie bracket. Having this projection we define the contact torsion $T: \Lambda^{2} H \rightarrow H$ of a partial contact connection $\nabla$ exactly as in Sect. 2.9.

Finally, there is a notion of contact projective equivalence for partial contact connections. Consider a smooth section $\Upsilon$ of the bundle $H^{*}$ of linear functionals on the contact subbundle. Then using the Levi bracket $\mathcal{L}$, we define $\tilde{\Upsilon}: Q \rightarrow H$ by $\mathcal{L}(\tilde{\Upsilon}(\tau), \eta)=\Upsilon(\eta) \tau$. Given a partial contact connection $\nabla$ on $H$, one defines a partial connection $\hat{\nabla}$ on $H$ by

$$
\hat{\nabla}_{\eta_{1}} \eta_{2}=\nabla_{\eta_{1}} \eta_{2}+\Upsilon\left(\eta_{1}\right) \eta_{2}+\Upsilon\left(\eta_{2}\right) \eta_{1}+\tilde{\Upsilon}\left(\mathcal{L}\left(\eta_{1}, \eta_{2}\right)\right) \text {. }
$$

One easily verifies directly that this is again a partial contact connection on $H$. Further, one shows that the projection $\hat{\pi}$ associated to $\hat{\nabla}$ is given by $\hat{\pi}(\zeta)=\pi(\zeta)+2 \tilde{\Upsilon}(\zeta+H)$ and this easily implies that $\hat{\nabla}$ and $\nabla$ have the same contact torsion.

One calls two partial contact connections contact projectively equivalent if they are related in the above way. A (torsion-free) contact projective structure on $M^{\#}$ is then given by a contact structure together with a class of contact projectively equivalent partial contact connections with vanishing contact torsion. It turns out that such a structure can be equivalently described by a Cartan geometry as discussed in Sect. 2.3, which corresponds to the contact grading of 
the Lie algebra $\mathfrak{s p}(2 n+2, \mathbb{R})$. As a group, one can take $G=S p(2 n+2, \mathbb{R})$ and $P \subset G$ the stabilizer of a ray in the standard representation $\mathbb{R}^{2 n+2}$ of $G$. Then it turns out that $G_{0}$ is the conformally symplectic group $\operatorname{CSp}(2 n, \mathbb{R})$, see Section 4.2 .6 of [7].

Remark 1 1. Similarly to classical projective structures, there is also an interpretation of contact projective equivalence in terms of geodesics in directions tangent to the contact distribution, and a corresponding description of contact projective structures, see [10] and Section 4.2.7 in [7].

2. In contrast to the case of usual affine connections, it is in general not possible to remove the contact torsion of a partial contact connection without changing its contact geodesics. Hence it is natural to extend the notion of a contact projective structure to the case of non-vanishing contact torsion, see [10]. It turns out that also in this more general setting, one can associate a canonical Cartan geometry to such a structure. The resulting Cartan geometry does not fit into the general scheme of regular normal parabolic geometries, however.

\subsection{Contactifications of conformally Fedosov structures}

Our final task in this article is to establish analogs of Theorems 2, 4 and 5 for contact projective structures and conformally Fedosov structures. This turns out to be rather easy since, as indicated in Sect. 3.1, the projective freedom in a conformally Fedosov structure does not really show up. Likewise, in the setting of contact projective structures, we will always have a preferred infinitesimal automorphism around. As discussed in Sect. 2.9, this determines a preferred Weyl structure and thus a distinguished representative in the contact projective class. Hence we can always relate connections rather than projective equivalence classes, and this works similarly as in the proof of Theorem 6.

Theorem 8 Let $q: M^{\#} \rightarrow M$ be a quotient of a contact manifold by a transverse infinitesimal contactomorphism $\xi \in \mathfrak{X}\left(M^{\#}\right)$, and let $\ell \subset \Lambda^{2} T^{*} M$ be the induced conformally symplectic structure.

1. A contact projective structure on $M^{\#}$, for which $\xi$ is an infinitesimal automorphism, induces a conformally Fedosov structure on $M$ with underlying conformally symplectic structure $\ell$.

2. Conversely, a conformally Fedosov structure on $M$ with underlying conformally symplectic structure $\ell$ canonically lifts to a contact projective structure for which $\xi$ is an infinitesimal automorphism.

3. Suppose that we have two conformally Fedosov structures realized as quotients of contact projective structures as in 2. Then any local lift of a diffeomorphism respecting the conformally Fedosov structures to a contactomorphism is automatically compatible with the infinitesimal automorphisms as in Theorem 5 and a local isomorphism of contact projective structures.

Proof Let $\mathcal{G}_{0}^{\#} \rightarrow M^{\#}$ be the natural frame bundle for the contact subbundle $H$ with structure group $G_{0}=\operatorname{CSp}(2 n, \mathbb{R})$ and let $\mathcal{G}_{0} \rightarrow M$ be the natural frame bundle for $T M$ induced by $\ell$, which also has structure group $G_{0}$, see Section 2.3 of [4]. By construction, there is a tautological soldering form $\theta \in \Omega^{1}\left(\mathcal{G}_{0}, \mathbb{R}^{2 n}\right)$. Denoting as before by $T^{-1} \mathcal{G}_{0}^{\#}$ the preimage of $H \subset T M^{\#}$ in $T \mathcal{G}_{0}^{\#}$, we likewise get a tautological soldering form $\theta_{-1}^{\#} \in \Gamma\left(L\left(T^{-1} \mathcal{G}_{0}^{\#}, \mathbb{R}^{2 n}\right)\right)$.

By definition, the local flows of $\xi$ are contactomorphisms and hence lift to local principal bundle automorphisms of $\mathcal{G}_{0}^{\#}$. Differentiating these local flows, we obtain a $G_{0}$-invariant lift 
$\xi_{0} \in \mathfrak{X}\left(\mathcal{G}_{0}^{\#}\right)$ of $\xi$. As observed in the proof of Theorem 2, the tangent maps of $q$ restrict to linear isomorphisms on the contact subspaces, which provides a $G_{0}$-equivariant lift $q_{0}: \mathcal{G}_{0}^{\#} \rightarrow \mathcal{G}_{0}$ of $q: M^{\#} \rightarrow M$. Moreover, by construction the restriction of $q_{0}$ to each fiber of $\mathcal{G}_{0}^{\#}$ is injective. As in the proofs of Lemma 1 and Theorem 2, this shows that $q_{0}$ is a surjective submersion and that the restriction of $p_{0}^{\#}: \mathcal{G}_{0}^{\#} \rightarrow M^{\#}$ to any fiber of $q_{0}$ is a diffeomorphism onto a fiber of $q$, so in particular $q_{0}$ has connected fibers. Finally, it is easy to see that the tangent spaces to these fibers are spanned by $\xi_{0}$.

1. We can pass to the Cartan geometry determined by the given contact projective structure on $M^{\#}$, which has $\mathcal{G}_{0}^{\#}$ as its underlying $G_{0}$-bundle. The general theory of Weyl structures applies to contact projective structures. Hence as in the proof of Theorem 6 , we see that the Weyl structure determined by $\xi$ determines a principal connection form $\tilde{\gamma}^{\#}$ on $\mathcal{G}_{0}^{\#}$. Here the Weyl connection is an extension of a partial contact connection with vanishing contact torsion, which lies in the contact projective class. Continuing as in the proof of Theorem 6, we can change this to a principal connection form $\gamma^{\#}$ for which $\xi_{0}$ is horizontal, and show that this descends to $\mathcal{G}_{0}$. Still as in that proof, we can verify that the resulting connection on $M$ is torsion-free and by construction it preserves $\ell$. So we can use the projective class of this connection to define a conformally Fedosov structure on $M$.

2. Starting with a conformally Fedosov structure on $M$, we know from Proposition 2 that there is a unique connection $\nabla$ in the projective class, which preserves the conformally symplectic structure $\ell$. Then $\nabla$ defines a principal connection $\gamma \in \Omega^{1}\left(\mathcal{G}_{0}, \mathfrak{g}_{0}\right)$. Now we can pull back this connection form to $\mathcal{G}_{0}^{\#}$ and then restrict it to $T^{-1} \mathcal{G}_{0}^{\#}$. The result is a $G_{0}$-equivariant section of $L\left(T^{-1} \mathcal{G}_{0}^{\#}, \mathfrak{g}_{0}\right)$ which by construction reproduces the generators of fundamental vector fields. Analogously to the standard construction of induced connections, this gives rise to a partial contact connection $\nabla^{\#}$ on $H \subset T M^{\#}$.

By construction, the local flows $\mathrm{Fl}_{t}^{\xi_{0}}$ satisfy $q_{0} \circ \mathrm{Fl}_{t}^{\xi_{0}}=q_{0}$ and they preserve the contact distribution $H \subset T M^{\#}$. This immediately implies that these flows preserve the pullback of $\gamma$ and its restriction to the contact distribution and hence the partial contact connection $\nabla^{\#}$. Now let $\alpha$ be the unique contact form on $M^{\#}$ such that $\alpha(\xi)=1$ and $\omega$ the unique symplectic form on $M$ in the given conformal symplectic class such that $q^{*} \omega=d \alpha$. Consider sections $\eta_{1}, \eta_{2}$ and $\zeta$ of $H \subset T M^{\#}$ which project onto vector fields $\underline{\eta}_{1}, \underline{\eta}_{2}$ and $\zeta$ on $M$. Then by definition, the Levi bracket $\mathcal{L}\left(\eta_{1}, \eta_{2}\right)$ is equal to $\alpha\left(\left[\eta_{1}, \eta_{2}\right]\right)(\bar{\xi}+\underline{H})=$ $-\left(\omega\left(\underline{\eta}_{1}, \underline{\eta}_{2}\right) \circ q\right)(\xi+H)$. Now by definition of the partial connection on $Q$ induced by $\nabla^{\#}$, we get

$$
\nabla_{\zeta}^{Q} \mathcal{L}\left(\eta_{1}, \eta_{2}\right)=\mathcal{L}\left(\nabla_{\zeta}^{\#} \eta_{1}, \eta_{2}\right)+\mathcal{L}\left(\eta_{1}, \nabla_{\zeta}^{\#} \eta_{2}\right)
$$

By construction $\nabla_{\zeta}^{\#} \eta_{i}$ is a lift of $\nabla_{\zeta} \underline{\eta}_{i}$ for $i=1,2$. From Proposition 2, we then know that $\nabla \omega=0$ and inserting this, we easily conclude that $\nabla^{Q}(\xi+H)=0$. Since $\xi$ is an infinitesimal contactomorphism, this implies that the projection $\pi: T M^{\#} \rightarrow H$ induced by $\nabla^{\#}$ satisfies $\pi(\xi)=0$, which of course determines $\pi$ completely.

Using these facts, we can now continue completely parallel to the proof of Theorem 6 . We see that $\pi\left(\left[\eta_{1}, \eta_{2}\right]\right) \in \Gamma(H)$ is the unique section of $H$ lifting $\left[\underline{\eta}_{1}, \underline{\eta}_{2}\right]$. Using this, torsion freeness of $\nabla$ implies that $\nabla^{\#}$ has vanishing contact torsion. Hence we can use its contact projective equivalence class to define a contact projective structure on $M$, and since the flows of $\xi$ even preserve the partial contact connection $\nabla^{\#}$, it is an infinitesimal automorphism of this structure. 
3. Let $\varphi: M \rightarrow \tilde{M}$ be the given morphism of conformally Fedosov structures and let $\varphi^{\#}: M^{\#} \rightarrow \tilde{M}^{\#}$ be a contactomorphism lifting $\varphi$. Then the first step of the proof of Theorem 5 shows that $\left(\varphi^{\#}\right)^{*} \tilde{\xi}=\lambda \xi$ for a nowhere vanishing, locally constant function $\lambda$. Now there are natural lifts $\Phi: \mathcal{G}_{0} \rightarrow \tilde{\mathcal{G}}_{0}$ and $\Phi^{\#}: \mathcal{G}_{0}^{\#} \rightarrow \tilde{\mathcal{G}}_{0}^{\#}$ to the frame bundles and $\left(\Phi^{\#}\right)^{*} \tilde{\xi}_{0}=\lambda \xi_{0}$. In view of the constructions of $\Phi^{\#}$ and $q_{0}$ and $\tilde{q}_{0}$, we see that $\tilde{q} \circ \varphi^{\#}=\varphi \circ q$ implies $\tilde{q}_{0} \circ \Phi^{\#}=\Phi \circ q_{0}$.

Now a morphism of conformally Fedosov structures preserves both the conformally symplectic structure and the projective class. Hence the uniqueness statement in statement (iii) in Proposition 2 implies that it is also compatible with the (unique) connections in the projective class which preserve the conformally symplectic structures. Hence for their connection forms, we get $\Phi^{*} \tilde{\gamma}=\gamma$, which together with the above implies that $\left(\Phi^{\#}\right)^{*} \tilde{q}_{0}^{*} \tilde{\gamma}=q_{0}^{*} \Phi^{*} \tilde{\gamma}=q_{0}^{*} \gamma$. Together with the fact that $\left(\Phi^{\#}\right)^{*} \tilde{\xi}_{0}=\lambda \xi_{0}$, this implies that $\Phi^{\#}$ is compatible with the partial contact connections constructed in (2) and hence in particular with the contact projective structures they generate.

Parallel to Theorem 7, there also is a relation between conformally Fedosov structure and a class of special symplectic connections. The relevant connections here are called symplectic connections of Ricci type, see [1] and [3]. The module of formal curvatures of symplectic connections splits over $\mathfrak{s p}(2 n)$ as a direct sum of the kernel of the Ricci-type contractions and a complementary submodule. A symplectic connection of Ricci type is a torsion-free symplectic connection for which the curvature is concentrated in that complementary submodule.

For a symplectic manifold $(M, \omega)$ and a torsion-free symplectic connection $\nabla$, the underlying conformally symplectic and projective structures evidently define a conformally Fedosov structure. On the other hand, for a general conformally Fedosov structure, the connection $\nabla$ from part (iii) of Proposition 2 has curvature in $\Lambda^{2} T^{*} M \otimes \mathfrak{s p}(T M)$ by the last part of the proposition. Hence the concept of being of Ricci type makes sense for this connection.

Corollary 1 The distinguished connection $\nabla$ of a conformally Fedosov structure from part (iii) of Proposition 2 is of Ricci type if and only if any local parabolic contactification of the structure is locally flat as a contact projective structure.

Proof Consider a local parabolic contactification $q: M^{\#} \rightarrow M$. In the construction of the contact projective structure on $M^{\#}$ in the proof of part 2 of Theorem 8 , we used $\nabla$ to construct a partial contact connection $\nabla^{\#}$ on $M^{\#}$. The verifications in that proof actually show that $\nabla^{\#}$ is the partial contact connection coming from the Weyl structure determined by the infinitesimal automorphism $\xi$ giving rise to $q$.

The parabolic geometry determined by a contact projective structure with vanishing contact torsion is well known to be torsion-free, see Section 4.2.6 of [7]. There it is also verified that the only harmonic curvature of a contact projective structure has homogeneity 2 and is a section of the bundle induces by the highest weight subspace in $\Lambda^{2} \mathfrak{g}_{-1}^{*} \otimes \mathfrak{g}_{0}$, which is exactly the kernel of the Ricci-type contraction. Now using Theorem 4.2.2 of [7], one concludes as in the proof of Theorem 7 that this harmonic curvature can be computed from $\nabla^{\#}$. Using the information from the proof of part 2 of Theorem 8 one deduces as in the proof of Theorem 7 that vanishing of the component is equivalent to vanishing of the corresponding component of the curvature of $\nabla$, which implies the result. 


\subsection{Example}

There is an analog of the global contactifications discussed in Sect. 2.6 in the context of conformally Fedosov structures. This actually is the example relevant for the results on integral geometry in [9] which motivate the developments in this series of articles.

Let us realize the standard symplectic form in dimension $2 n+2$ as the imaginary part of the standard Hermitian form on $\mathbb{C}^{n+1}$. This gives rise to an action of $G=\operatorname{Sp}(2 n+2, \mathbb{R})$ on $\mathbb{C}^{n+1}$ (which does not preserve the complex structure). Viewing $S^{2 n+1}$ as the space of real rays in $\mathbb{C}^{n+1}$ we get a transitive action of $G$, which makes $S^{2 n+1}$ into the homogeneous model for contact projective structures, compare with Section 4.2.6 of [7]. Now the standard action of $U(1)$ on $\mathbb{C}^{n+1}$ by complex multiplication defines a subgroup in $G$ and hence an action of $U(1)$ on $S^{2 n+1}$ by automorphisms of the contact projective structure. The tangent space of $S^{2 n+1}$ in a point corresponding to a ray in $\mathbb{C}^{n+1}$ can be viewed as the quotient of the real vector space $\mathbb{C}^{n+1}$ by the line spanned by the ray. The contact subspaces then correspond to the quotient of the symplectic orthocomplement of the ray by this line. This readily implies that the infinitesimal generator of our $U(1)$-action is transversal everywhere. Of course the $U(1)$-action on $S^{2 n+1}$ admits a global quotient, namely the canonical projection $q: S^{2 n+1} \rightarrow \mathbb{C} P^{n}$ mapping a real ray in $\mathbb{C}^{n+1}$ to the complex line it spans.

Hence we obtain exactly the same quotient map as in the first example in Sect. 2.6, where we started from the CR-structure on $S^{2 n+1}$, which is also preserved by the $U(1)$-action. It is actually easy to see that the Weyl connections associated to the infinitesimal generator of the $U(1)$-action via the CR-structure and via the projective contact structure coincide. But this implies that the underlying conformally Fedosov structure on $\mathbb{C} P^{n}$ actually is the one determined by the Levi-Civita connection of its Kähler metric.

Remark 2 The last bit of this example actually exhibits a general phenomenon. Suppose that we have a torsion-free PCS-structure (not of type $C_{n}$ ) on a smooth manifold $M$. Then via Proposition 2, the (torsion-free) canonical connection actually determines an underlying conformally Fedosov structure. This may look surprising, since there is no corresponding construction for an "underlying contact projective structure" associated to a (torsion-free) parabolic contact structure of different type. However, such an underlying structure does become available in the presence of a transversal infinitesimal automorphism. Indeed, one can then consider the Weyl connection determined by this transversal infinitesimal automorphism and restrict it to a partial contact connection. General results then ensure that in the case of a torsion-free parabolic contact structure, this partial contact connection has vanishing contact torsion and hence determines a torsion-free contact projective structure, which is preserved by the given infinitesimal automorphism. Hence a contactification of the initial PCS-structure at the same time defines a contactification of the underlying conformally Fedosov structure.

Acknowledgements Open access funding provided by Austrian Science Fund (FWF).

Open Access This article is distributed under the terms of the Creative Commons Attribution 4.0 International License (http://creativecommons.org/licenses/by/4.0/), which permits unrestricted use, distribution, and reproduction in any medium, provided you give appropriate credit to the original author(s) and the source, provide a link to the Creative Commons license, and indicate if changes were made.

\section{References}

1. Bourgeois, F., Cahen, M.: A variational principle for symplectic connections. J. Geom. Phys 30(3), 233265 (1999). https://doi.org/10.1016/S0393-0440(98)00059-X 
2. Bryant, R.L., Chern, S.S., Gardner, R.B., Goldschmidt, H.L., Griffiths, P.A.: Exterior Differential Systems, Mathematical Sciences Research Institute Publications, vol. 18. Springer, New York (1991)

3. Cahen, M., Schwachhöfer, L.J.: Special symplectic connections. J. Differ. Geom. 83(2), 229-271 (2009)

4. Čap, A., Salač, T.: Parabolic Conformally Symplectic Structures I: Definition and Distinguished Connections, Forum Math., to appear. arxiv:1605.01161. https://doi.org/10.1515/forum-2017-0018

5. Čap, A., Salač, T.: Parabolic Conformally Symplectic Structures III: Invariant Differential Operators and Complexes. arXiv: 1701.01306

6. Čap, A., Salač, T.: Pushing down the Rumin complex to conformally symplectic quotients. Differ. Geom. Appl. 35, 255-265 (2014). https://doi.org/10.1016/j.difgeo.2014.05.004

7. Čap, A., Slovák, J.: Parabolic Geometries. I, volume 154 of Mathematical Surveys and Monographs. Background and General Theory. American Mathematical Society, Providence (2009)

8. Eastwood, M.G., Slovák, J.: Conformally Fedosov manifolds. arXiv:1210.5597

9. Eastwood, M., Goldschmidt, H.: Zero-energy fields on complex projective space. J. Differ. Geom. 94(1), 129-157 (2013)

10. Fox, D.J.F.: Contact projective structures. Indiana Univ. Math. J 54(6), 1547-1598 (2005). https://doi. org/10.1512/iumj.2005.54.2603

11. Kostant, B.: Lie algebra cohomology and the generalized Borel-Weil theorem. Ann. Math. 74(2), 329-387 (1961)

12. Sternberg, S.: Lectures on differential geometry. Prentice-Hall Inc., Englewood Cliffs (1964) 